\title{
DENOMINATOR IDENTITY FOR TWISTED AFFINE LIE SUPERALGEBRAS
}

\author{
SHIFRA REIF
}

\begin{abstract}
The study of denominator identities for Lie superalgebras was recently developed by M. Gorelik, V.G. Kac, P.Moseneder Frajria, I. Musson, P. Papi, M. Wakimoto and the author. In this paper we generalize these identities to the twisted affine case, completing the result to all affine Lie superalgebras.
\end{abstract}

\section{INTRODUCTION}

The study of denominator identities for Lie superalgebras was initiated by V.G. Kac and M. Wakimoto in $[\mathrm{KW}$. These identities were shown to have interesting applications to number theory (see $[\mathrm{KW}, \mathrm{M}, \mathbf{Z}]$ ), vacuum modules and minimal $W$-algebras (see [HR, GK]) and the Howe duality for compact dual pairs (see $[\mathrm{FKP}$ ).

The denominator identities for basic Lie superalgebras and (non-twisted) affine Lie superalgebras with non-zero dual Coxeter number were formulated and partially proven by V.G. Kac and M. Wakimoto [KW]. Complete proofs were given by M. Gorelik in [G1, G2]. The denominator identity for the strange $\hat{Q}$ series was conjectured in $[\mathrm{KW}$ ] and proven by D. Zagier in [Z using analytic methods. The case where the dual Coxeter number is zero was proven in [GR].

For a semisimple Lie algebra, the denominator identity follows by applying the Weyl character formula to the trivial representation and takes the form $e^{\rho} R=\sum_{w \in W}(\operatorname{sgn} w) w e^{\rho}$ where $R:=$ $\prod_{\alpha \in \Delta^{+}}\left(1-e^{-\alpha}\right)$ is the denominator of $\mathfrak{g}$ corresponding to the positive roots $\Delta^{+}, W$ the affine Weyl group and $\rho$ is chosen such that $(\rho, \alpha)=\frac{1}{2}(\alpha, \alpha)$ for every simple root $\alpha$. Similarly, we have the identity $e^{\hat{\rho}} \hat{R}=\sum_{w \in \hat{W}}(\operatorname{sgn} w) w e^{\hat{\rho}}$ for the an affine algebra $\hat{\mathfrak{g}}$. The affine Weyl group $\hat{W}$ has a decomposition $\hat{W}=T \rtimes W$ where $W$ corresponds to one of the maximal finite subalgebra of $\hat{\mathfrak{g}}$ and $T$ is an abelian group of translations. Since $\hat{\rho}-\rho$ is $W$-invariant and the elements of $T$ are of sign 1 , we can rewrite the denominator identity as

$$
e^{\hat{\rho}} \hat{R}=\sum_{t \in T} t\left(e^{\hat{\rho}} R\right)=\sum_{t \in T}(\operatorname{sgn} t) t\left(e^{\hat{\rho}} R\right) .
$$

In this paper we prove the denominator identity for twisted affine Lie superalgebras by generalizing the latter formula. In vdL1, vdL2, V. de Leur classified these algebras and showed that all symmetrizable Kac Moody superalgebras of finite growth are either finite, affine or twisted affine. The classification consists of the series: $A(2 k-1,2 l-1)^{(2)}, A(2 k, 2 l-1)^{(2)}, A(2 k, 2 l)^{(4)}$, $C(k+1)^{(2)}$ and $D(k+1, l)^{(2)}$ and the exceptional Lie superalgebra $G(3)^{(2)}$. The description of the automorphisms and the root systems given in vdL2 is summarized in Appendix 6.1.

Let $\hat{\mathfrak{g}}=\tilde{\mathfrak{g}}^{(m)}$ be a twisted affine Lie superalgebras defined by an automorphism of order $m$ and $\mathfrak{g}$ the algebra formed by the fixed points under this automorphism. Let $\hat{R}$ and $R$ be the denominators of $\hat{\mathfrak{g}}$ and $\mathfrak{g}$ (see formulas (2.1), (2.2)), respectively, and $h^{\vee}:=(\hat{\rho}, \delta)$ the dual Coxeter number where

Supported by the Minerva foundation with funding from the Federal German Ministry for Education and Research. 
$\delta$ is the minimal imaginary root (see 2.1). For $\hat{\mathfrak{g}} \neq A(2 k-1,2 l-1)^{(2)}, k \geq l$, we take $T^{\prime}$ to be the translation group that corresponds to the "larger" part of the Weyl group when $h^{\vee} \neq 0$ and to the "smaller" part when $h^{\vee}=0$ (see 2.3). For $\hat{\mathfrak{g}}=A(2 k-1,2 l-1)^{(2)}, k \geq l$, we extend the affine Weyl group by a diagram automorphism and take $T^{\prime}$ to be the translation group corresponding to this extension. The sign function is extended by setting this diagram automorphism to be of sign 1.

We prove the following theorem:

Theorem 1.1. Let $q:=e^{-\delta}$. Then the following identity holds:

$$
e^{\hat{\rho}} \hat{R}=f(q) \cdot \sum_{t \in T^{\prime}}(\operatorname{sgn} t) t\left(e^{\hat{\rho}} R\right)
$$

where

$$
f(q)=\left\{\begin{array}{cc}
1 & \text { when } h^{\vee} \neq 0 \\
\prod_{n=1}^{\infty}\left(1-q^{2 n+1}\right)^{-2} & A(2 k-1,2 k-1)^{(2)} \\
\prod_{n=1}^{\infty}\left(1+q^{2 n+1}\right)^{-1} & A(2 k, 2 k)^{(4)} \\
\prod_{n=1}^{\infty}\left(1-q^{2 n+1}\right) & D(k+1, k)^{(2)} .
\end{array}\right.
$$

We extend the proofs of the non-twisted cases in G1, GR. The proof splits into two parts, namely the cases of dual Coxeter number zero and non-zero. We use the action of the Casimir operator as well as the denominator identity for basic Lie superalgebras. As $e^{\hat{\rho}} \hat{R}$ is independent of the choice of simple roots, we prove the identity for convenient choices described in Appendix 6.2.

Acknowledgments. The paper is based on a part of the author's dissertation done under the supervision of Prof. M. Gorelik. The author would like to thank M. Gorelik for reading several drafts of this paper, suggesting ways to improve and fix it. The author is also grateful for A. Joseph for helpful conversations and to G. Binyamini, C. Hoyt and X. Lamprou for discussing on the presentation of this paper.

\section{Preliminaries}

We introduce standard notations and elementary facts that are used in the paper.

2.1. Twisted affine Lie superalgebras. Let $\tilde{\mathfrak{g}}$ be a basic simple Lie superalgebra with nondegenerate invariant bilinear form $(\cdot, \cdot)$ and $\sigma$ an automorphism of finite order $m>1$. The eigenvalues of $\sigma$ are of the form $e^{\frac{2 \pi i}{m} k}, k \in \mathbb{Z}_{m}$ and hence $\tilde{\mathfrak{g}}$ admits the following $\mathbb{Z}_{m}$-grading:

$$
\tilde{\mathfrak{g}}=\bigoplus_{k \in \mathbb{Z}_{m}} \tilde{\mathfrak{g}}_{(k)}, \quad \tilde{\mathfrak{g}}_{(k)}=\left\{x \in \tilde{\mathfrak{g}} \mid \sigma(x)=e^{\frac{2 \pi i}{m} k} \cdot x\right\}
$$

The twisted affine Lie superalgebra is defined to be

$$
\hat{\mathfrak{g}}:=\left(\bigoplus_{k \in \mathbb{Z}_{m}} \mathbb{C} t^{k} \otimes \tilde{\mathfrak{g}}_{(k(\bmod m))}\right) \oplus \mathbb{C} K \oplus \mathbb{C} D
$$

with the relations

$$
\left[t^{i} \otimes g_{1}, t^{j} \otimes g_{2}\right]=t^{i+j} \otimes\left[g_{1}, g_{2}\right]+i \delta_{i,-j}\left(g_{1}, g_{2}\right) K, \quad[\hat{\mathfrak{g}}, K]=0, \quad\left[D, t^{i} \otimes g\right]=i t^{i} \otimes g .
$$

The fixed points of $\sigma$ form a maximal finite subalgebra of $\hat{\mathfrak{g}}$ which we denote by $\mathfrak{g}$.

Let $\mathfrak{h}$ be a Cartan subalgebra of $\mathfrak{g} \subset \tilde{\mathfrak{g}}$ and $\tilde{\mathfrak{g}}=\bigoplus_{\alpha \in \mathfrak{h}^{*}} \tilde{\mathfrak{g}}_{\alpha}$ a decomposition where

$$
\tilde{\mathfrak{g}}_{\alpha}:=\{g \in \tilde{\mathfrak{g}} \mid[h, g]=\alpha(h) \cdot g, \forall h \in \mathfrak{h}\} .
$$


For every $i=0,1, j=0, \ldots m-1$, let

$$
\Delta_{\bar{i}}^{(j)}:=\left\{\alpha \in \mathfrak{h}^{*} \mid \tilde{\mathfrak{g}}_{\alpha} \cap \tilde{\mathfrak{g}}_{(j)} \cap \tilde{\mathfrak{g}}_{\bar{i}} \neq\{0\}\right\}
$$

be a multi-set such that the multiplicity of $\alpha$ is $\operatorname{dim} \tilde{\mathfrak{g}}_{\alpha} \cap \tilde{\mathfrak{g}}_{(j)} \cap \tilde{\mathfrak{g}}_{\bar{i}}$. We get that the set of roots of $\mathfrak{g}$ is $\Delta=\Delta_{\overline{0}} \cup \Delta_{\overline{1}}$ where $\Delta_{\bar{i}}:=\Delta_{\bar{i}}^{(0)}$. The set of roots $\hat{\Delta}$ of $\hat{\mathfrak{g}}$ takes the form $\hat{\Delta}=\hat{\Delta}_{\overline{0}} \cup \hat{\Delta}_{\overline{1}}$ where

$$
\hat{\Delta}_{\bar{i}}=\left\{l \delta+\alpha \mid \alpha \in \Delta_{\bar{i}}^{(j)}, l=j(\bmod m) \text { such that } l \delta+\alpha \neq 0\right\} .
$$

Note that the sets $\Delta_{\bar{i}}^{(j)}$ may contain 0 , in which case the imaginary roots $l \delta \in \hat{\Delta}$ can be either odd or even.

Fix a set $\pi$ of simple roots of $\mathfrak{g}$. We take $\hat{\pi}=\left\{\alpha_{0}:=\delta-\theta\right\} \cup \pi$ to be the set of simple roots of $\hat{\mathfrak{g}}$, where $\theta$ is the highest weight in $\Delta_{\overline{0}}^{(1)} \cup \Delta_{\overline{1}}^{(1)}$. The root lattice of $\hat{\mathfrak{g}}$ is defined to be $\hat{Q}=\sum_{i=0}^{n} \mathbb{Z} \alpha_{i}$. Let $\hat{Q}^{+}=\sum_{i=0}^{n} \mathbb{N} \alpha_{i}$. Define the partial ordering on $\hat{\mathfrak{h}}^{*}$ by $\mu \geq \nu$ if $\mu-\nu \in \hat{Q}^{+}$. We extend $(\cdot, \cdot)$ to $\hat{\mathfrak{g}}$ in the standard way. Let $\rho \in \mathfrak{h}^{*}$ be such that $(\rho, \alpha)=\frac{1}{2}(\alpha, \alpha)$ for every $\alpha$ in $\pi$. Let $\Lambda_{0} \in \hat{\mathfrak{h}}^{*}$ be such that $\left(\Lambda_{0}, \delta\right)=1$ and $\left(\Lambda_{0}, \Lambda_{0}\right)=\left(\Lambda_{0}, \mathfrak{h}\right)=0$. Let $\hat{\rho}:=h^{\vee} \Lambda_{0}+\rho$.

Let $\hat{\Delta}_{\overline{0}}^{+}$and $\hat{\Delta}_{\overline{1}}^{+}$be the positive even and odd roots of $\hat{\mathfrak{g}}$, respectively and $\Delta_{\overline{0}}^{+}$and $\Delta_{\overline{1}}^{+}$the positive even and odd roots of $\mathfrak{g}$, respectively. The denominators of $\hat{\mathfrak{g}}$ and $\mathfrak{g}$ are defined to be

$$
\begin{array}{ll}
R:=\frac{R_{\overline{0}}}{R_{\overline{1}}}, \quad R_{\overline{0}}=\prod_{\alpha \in \Delta_{\overline{0}}^{+}}\left(1-e^{-\alpha}\right) \text { and } R_{\overline{1}}=\prod_{\alpha \in \Delta_{\overline{1}}^{+}}\left(1+e^{-\alpha}\right) \\
\hat{R}:=\frac{\hat{R}_{\overline{0}}}{\hat{R}_{\overline{1}}}, \quad \hat{R}_{\overline{0}}=\prod_{\alpha \in \hat{\Delta}_{\overline{0}}^{+}}\left(1-e^{-\alpha}\right) \text { and } \hat{R}_{\overline{1}}=\prod_{\alpha \in \hat{\Delta}_{\overline{1}}^{+}}\left(1+e^{-\alpha}\right) .
\end{array}
$$

Recall that $q=e^{-\delta}$. The affine denominator takes the form

$$
\hat{R}=R \cdot \prod_{n=1}^{\infty} \prod_{j=0}^{m-1} \frac{\prod_{\alpha \in \Delta_{\overline{0}}^{(j)}}\left(1-q^{m n+j} e^{-\alpha}\right)}{\prod_{\alpha \in \Delta_{\overline{1}}^{(j)}}\left(1+q^{m n+j} e^{-\alpha}\right)}
$$

where the elements from $\Delta_{\bar{i}}^{(j)}$ are taken with multiplicity.

2.2. The Weyl group of a Lie superalgebra. One of the main tools that is used in the proof is the action of certain subgroups of the Weyl group. We recall the definition of the Weyl group for twisted affine Lie superalgebras and some facts about its action.

An even root $\alpha$ is called principal if in some base $\Pi^{\prime}$ obtained from $\pi$ by a sequence of odd reflections, either $\alpha$ or $\frac{\alpha}{2}$ is simple (see [HS, 5]). The Weyl group is defined to be the group generated by reflections with respect to the principal roots. For basic simple Lie superalgebras, it coincides with the group generated by reflections with respect to the even roots. For twisted affine Lie superalgebra, the real even roots can be identified with the real roots of a Kac-Moody algebra and the Weyl group is generated by these roots (see $[\mathrm{S}]$ ).

We shall use the following lemma in the proof of Theorem 1.1 for the case $h^{\vee} \neq 0$.

Lemma 2.1. (G1, 1.3.2]) Let $\Pi_{+}$be the set of principal roots satisfying $\left\langle\hat{\rho}, \alpha^{\vee}\right\rangle \geq 0$ for all $\alpha \in \Pi_{+}$, and $W_{+}$the subgroup of the Weyl group generated by the reflections $\left\{s_{\alpha} \mid \alpha \in \Pi_{+}\right\}$. Then

(i) One has $\hat{\rho}-w \hat{\rho} \in \hat{Q}^{+}$for all $w \in W_{+}$.

(ii) If $w=s_{\alpha_{i_{1}}} \cdot \ldots \cdot s_{\alpha_{i_{r}}}$ is reduced decomposition of $w \in W_{+}$, then

$$
h t(\hat{\rho}-w \hat{\rho}) \geq\left|\left\{j \mid\left\langle\hat{\rho}, \alpha_{i_{j}}^{\vee}\right\rangle \neq 0\right\}\right| .
$$


(iii) The stabilizer of $\hat{\rho}$ in $W_{+}$is generated by the reflections $\left\{s_{\alpha} \mid \alpha \in \Pi_{+}\right.$and $\left.\left\langle\hat{\rho}, \alpha^{\vee}\right\rangle=0\right\}$.

2.3. The translation group $T^{\prime}$ and the denominator identity for basic Lie superalgebras. We define the subgroups of the Weyl group including $T^{\prime}$ that are used in the proof of Theorem 1.1 and normalize the bilinear form. We shall then recall the denominator identity for basic Lie superalgebras.

Definition 2.2. Let $\Delta_{1}$ and $\Delta_{2}$ be irreducible finite root systems of Lie algebras. We say that $\Delta_{1}$ is larger than $\Delta_{2}$ if its rank is larger, or if the ranks are equal and $\Delta_{2}$ can be embedded in $\Delta_{1}$.

For a basic simple Lie superalgebra (excluding $D(2,1, \alpha)$ ), the set of even roots $\Delta_{\overline{0}}$ is a root system of a reductive Lie algebra which is a product of at most two irreducible root systems. Let $\Delta^{\#}$ be the larger among the two and $W^{\#}$ its Weyl group. If $h^{\vee} \neq 0$, we take $\Delta^{\prime}$ (resp. $\Delta^{\prime \prime}$ ) to be the larger (resp. smaller) simple root subsystem and conversely otherwise. If the two simple parts are isomorphic, we pick an arbitrary choice. When they are incomparable $\left(C_{n}\right.$ and $\left.B_{n}\right)$, we shall specify for each case. For $D(2,1, \alpha), \Delta_{\overline{0}}=A_{1} \sqcup A_{1} \sqcup A_{1}$ and we take $\Delta^{\prime}=\Delta^{\#}=A_{1} \sqcup A_{1}$, $\Delta^{\prime \prime}=A_{1}$.

We normalize the bilinear form such that it is positive definite on $\Delta^{\prime}$. Let $\hat{\Delta}^{\prime}$ (resp. $\hat{\Delta}^{\prime \prime}$ ) be the maximal affine root subsystem of $\hat{\Delta}_{\overline{0}}$ with containing $\Delta^{\prime}$ (resp. $\Delta^{\prime \prime}$ ). The intersection $\hat{\Delta}^{\prime+}:=\hat{\Delta}^{\prime} \cap \hat{\Delta}^{+}$is a choice of positive roots for $\hat{\Delta}^{\prime}$. Let $\hat{\pi}^{\prime}$ be the corresponding set of simple roots and choose $\hat{\rho}^{\prime} \in \operatorname{span}\left(\hat{\pi}^{\prime} \cup\left\{\Lambda_{0}\right\}\right)$ such that $\left(\hat{\rho}^{\prime}, \alpha\right)=\frac{1}{2}(\alpha, \alpha)$ for every $\alpha \in \hat{\pi}^{\prime}$. The root lattice of $\hat{\Delta}^{\prime}$ is $\hat{Q}^{\prime}:=\mathbb{Z} \hat{\Delta}^{\prime}$. Let $\pi^{\prime \prime}$ be the set of simple roots of $\Delta^{\prime \prime}$ with respect to $\Delta^{\prime \prime+}:=\Delta^{\prime \prime} \cap \Delta^{+}$.

Let $W^{\prime}$ and $\hat{W}^{\prime}$ be the Weyl group of $\Delta^{\prime}$ and $\hat{\Delta}^{\prime}$, respectively. Let $M^{\prime}$ be the lattice generated by $\left\{\frac{2 n}{(\alpha, \alpha)} \alpha \mid n \delta-\alpha \in \hat{\Delta}^{\prime}, \alpha \in \mathfrak{h}^{*}\right\}$ and $T^{\prime}$ the abelian group generated by the translations

$$
\left\{t_{\alpha}(\lambda)=\lambda+(\lambda, \delta) \alpha-\left((\lambda, \alpha)+\frac{1}{2}(\alpha, \alpha)(\lambda, \delta)\right) \delta \mid \alpha \in M^{\prime}, \lambda \in \mathfrak{h}^{*}\right\} .
$$

Note that $t_{\frac{2 n}{(\alpha, \alpha)} \alpha}=s_{n \delta-\alpha} s_{\alpha}$. Unless $\hat{W}^{\prime}$ is of type $A_{2 n-1}^{(2)}$ and $W^{\prime}$ is of type $D_{k}$, we have that $T^{\prime} \subset \hat{W}^{\prime}$ (since in these cases $n \delta-\alpha \in \hat{\Delta}^{\prime}$ implies that $\alpha \in \mathbb{Q} \Delta^{\prime}$ ) and $\hat{W}^{\prime}=W^{\prime} \ltimes T^{\prime}$ (see [K] 6.5]).

When $\hat{W}^{\prime}$ is of type $A_{2 n-1}^{(2)}$ and $W^{\prime}$ is of type $D_{k}$ (that is, when $\hat{\mathfrak{g}}=A(2 k-1,2 l-1)^{(2)}, k \geq l$ ), we have that $\Delta^{\prime}=\left\{\varepsilon_{i} \pm \varepsilon_{j}\right\}$ and $M=\operatorname{span}_{\mathbb{Z}}\left\{\varepsilon_{1}, \ldots, \varepsilon_{n}\right\}$. Let $W_{C_{k}}$ and $\hat{W}_{C_{k}}$ be the Weyl groups of $C_{k}$ and $\hat{C}_{k}$, respectively. Note that $W_{C_{k}}=\left\langle W^{\prime}, s_{\varepsilon_{k}}\right\rangle, \hat{W}_{C_{k}}=\left\langle\hat{W}^{\prime}, s_{\varepsilon_{k}}\right\rangle$ and $s_{\varepsilon_{k}}$ corresponds to a diagram automorphism of $\Delta^{\prime}$. One has $\hat{W}_{C_{k}}=T^{\prime} \rtimes W_{C_{k}}$. We can check that the sign function can be extended from $\hat{W}^{\prime}$ to $\hat{W}_{C_{k}}$ by setting $\operatorname{sgn} s_{\varepsilon_{k}}=1$ (that is, $\operatorname{sgn} t_{\varepsilon_{k}}=-1$ ). The bilinear form $(\cdot, \cdot)$ is invariant under diagram automorphisms and thus under $\hat{W}_{C_{k}}$. A similar idea for extending the Weyl group was used in [FKP, 7].

We take $W^{\prime \prime}$ and $\hat{W}^{\prime \prime}$ to be the Weyl groups of $\Delta^{\prime \prime}$ and $\hat{\Delta}^{\prime \prime}$, respectively. Define $M^{\prime \prime}$ as $M^{\prime}$ (replacing $\hat{\Delta}^{\prime}$ with $\hat{\Delta}^{\prime \prime}$ ) and $T^{\prime \prime}$ as we defined $T^{\prime}$ (replacing $M^{\prime}$ with $M^{\prime \prime}$ ).

The defect of $\mathfrak{g}$ is the dimension of a maximal isotropic subspace of $\mathfrak{h}_{\mathbb{R}}^{*}:=\sum_{\alpha \in \Delta} \mathbb{R} \alpha$. A subset $S \subset \Delta_{1}^{+}$is called isotropic if it spans an isotropic subspace and maximal isotropic if moreover $|S|$ is equal to the defect. Given a maximal isotropic set $S$, there exists a choice of simple roots $\pi$ such that $S \subset \pi([\mathrm{KW}, 2.2])$. Thus, for the rest of the paper we shall assume that our choice of simple roots contains a maximal isotropic set.

Within this setup, recall that the denominator identity for basic Lie superalgebras, proven in [KW, G2], takes the following form: 


$$
e^{\rho} R=\sum_{w \in W^{\#}}(\operatorname{sgn} w) w\left(\frac{e^{\rho}}{\prod_{\beta \in S}\left(1+e^{-\beta}\right)}\right) .
$$

2.4. Algebras of formal power series. We recall the definition of the algebra of formal power series in which the equality of Theorem 1.1 holds (see also [G1, 1.4]).

Definition 2.3. Let $\mathcal{R}$ be the $\mathbb{Q}$-vector space spanned by the sums of the form $\sum_{\nu \in \hat{Q}^{+}} b_{\nu} e^{\lambda-\nu}$ where $\lambda \in \hat{\mathfrak{h}}^{*}$ and $b_{\nu} \in \mathbb{Q}$. For $Y:=\sum_{\nu \in \hat{\mathfrak{h}}^{*}} b_{\nu} e^{\nu} \in \mathcal{R}$, we define the support of $Y$ to be

$$
\operatorname{supp}(Y):=\left\{\nu \in \hat{\mathfrak{h}}^{*} \mid b_{\nu} \neq 0\right\} .
$$

Note that the ring $\mathcal{R}$ is not closed under the action of the Weyl group. Let $\tilde{W}$ be a subgroup of the Weyl group. We define subrings $\mathcal{R}_{\tilde{W}}$ and $\mathcal{R}^{\prime}$ of $\mathcal{R}$ which are closed under the action of $\tilde{W}$.

Let $\mathcal{R}_{\tilde{W}}$ be the subalgebra of $\mathcal{R}$ defined by

$$
\mathcal{R}_{\tilde{W}}:=\left\{\sum_{\nu \in \hat{\mathfrak{h}}^{*}} b_{\nu} e^{\nu} \in \mathcal{R} \mid \sum_{\nu \in \hat{\mathfrak{h}}^{*}} b_{\nu} e^{w \nu} \in \mathcal{R} \text { for all } w \in \tilde{W}\right\}
$$

and $\mathcal{R}^{\prime}$ the localization of $\mathcal{R}_{\tilde{W}}$ by

$$
\mathcal{Y}:=\left\{\prod_{\alpha \in X}\left(1+a_{\alpha} e^{-\alpha}\right)^{r(\alpha)} \mid a_{\alpha} \in \mathbb{Q}, r(\alpha) \in \mathbb{Z}_{\geq 0} \text { and } X \subset \hat{\Delta},\left|X \backslash \hat{\Delta}^{+}\right|<\infty\right\} .
$$

The elements of $\mathcal{Y}$ are invertible in $\mathcal{R}$ using geometric series (for example $\left(1-e^{-\alpha}\right)^{-1}=-e^{-\alpha}\left(1-e^{\alpha}\right)^{-1}$ $=-\sum_{i=1}^{\infty} e^{i \alpha}$ ) and $\mathcal{Y}$ is contained in $\mathcal{R}_{\tilde{W}}$ (see [G1, 1.4.2]). We extend the action of $\tilde{W}$ from $\mathcal{R}_{\tilde{W}}$ to $\mathcal{R}^{\prime}$ by $w\left(Y^{-1} Y^{\prime}\right)=(w Y)^{-1}\left(w Y^{\prime}\right)$ (see [G1, 1.4.3]).

Remark 2.4. Note that the maximal element in the support of $\prod_{\alpha \in X}\left(1+a_{\alpha} e^{-\alpha}\right)^{r(\alpha)} \in \mathcal{Y}$ is $-\sum_{\alpha \in X \backslash \hat{\Delta}^{+}: a_{\alpha} \neq 0} r(\alpha) \nu$.

For $Y \in \mathcal{R}_{\tilde{W}}$ such that each $\tilde{W}$-orbit in $\hat{\mathfrak{h}}^{*}$ has a finite intersection with $\operatorname{supp} Y$, denote the sum

$$
\mathcal{F}_{\tilde{W}}(Y):=\sum_{w \in \tilde{W}} \operatorname{sgn}(w) w Y .
$$

We use the following lemmas from [G1, 1.4.4]:

Lemma 2.5. Suppose $Y \in \mathcal{R}_{\tilde{W}}$ and $\mathcal{F}_{\tilde{W}}(Y) \in \mathcal{R}$, then $\mathcal{F}_{\tilde{W}}(Y) \in \mathcal{R}_{\tilde{W}}$ and is $\tilde{W}$-anti-invariant

A set is called $\tilde{W}$-regular if for every element $\lambda$ in the set, $\operatorname{Stab}_{\tilde{W}} \lambda$ is trivial.

Lemma 2.6. The support of a $\tilde{W}$-anti-invariant element in $\mathcal{R}_{\tilde{W}}$ is a union of regular $\tilde{W}$-orbits.

Remark 2.7. In order to use the above lemmas for $\hat{R}$ and $\mathcal{F}_{T^{\prime}}\left(e^{\hat{\rho}} R\right) \in \mathcal{R}^{\prime}$, we will multiply them by $\hat{R}_{1} \in \mathcal{Y}$. Note that since $e^{\hat{\rho}} \hat{R}$ and $e^{\hat{\rho}^{\prime}} \hat{R}_{\overline{0}}$ are $\hat{W}^{\prime}$-skew invariant elements of $\mathcal{R}_{\hat{W}^{\prime}}, e^{\hat{\rho}^{\prime}-\hat{\rho}} \hat{R}_{1}$ is $\hat{W}^{\prime}$-invariant. Similarly $e^{\hat{\rho}^{\prime \prime}-\hat{\rho}} \hat{R}_{1}$ is $\hat{W}^{\prime \prime}$-invariant. 


\section{MAIN ARGUMENT}

In this section we use the Casimir operator and the denominator identity for finite dimensional Lie superalgebras to show that the supports of $e^{\hat{\rho}} \hat{R}$ and $\mathcal{F}_{T^{\prime}}\left(e^{\hat{\rho}} R\right)$ belong to the following subset of $\hat{\mathfrak{h}}^{*}$ :

$$
U:=\left\{\mu \in \hat{\mathfrak{h}}^{*} \mid(\mu, \mu)=(\hat{\rho}, \hat{\rho})\right\} .
$$

Let us explain how this argument is used to prove Theorem 1.1 First, using $\hat{W}^{\prime}$-anti-invariance (namely lemmas 2.5 and 2.6), we get that it is sufficient to check the denominator identity only on a small subset of the coefficients. The fact that these are coefficients of elements in $U$, implies that one has to check the identity on even fewer coefficients. When $h^{\vee} \neq 0$, the rest of the proof amounts to comparing the coefficient of $e^{\hat{\rho}}$ on both sides of the identity. When $h^{\vee}=0$, one should calculate the coefficients of the powers of $q$. This is carried out in sections 4 and 5 respectively.

We shall use the following classical lemma (see for example [K, 10.4]).

Lemma 3.1. One has $\operatorname{supp}\left(e^{\hat{\rho}} \hat{R}\right) \subset U$.

Proof. Since $\hat{\mathfrak{g}}$ admits a Casimir element, the character of the trivial $\hat{\mathfrak{g}}$-module is an integral linear combination of the characters of Verma $\hat{\mathfrak{g}}$-modules $M(\lambda)$, where $\lambda \in-\hat{Q}^{+}$, are such that $(\lambda+\hat{\rho}, \lambda+\hat{\rho})=(\hat{\rho}, \hat{\rho})$ (see [K, 9.8]). Since the character of $M(\lambda)$ is equal to $\hat{R}^{-1} e^{\lambda}$, we obtain

$$
1=\sum_{\lambda \in-\hat{Q}^{+},(\lambda+\hat{\rho}, \lambda+\hat{\rho})=(\hat{\rho}, \hat{\rho})} a_{\lambda} \operatorname{ch} M(\lambda)=\sum_{\lambda \in-\hat{Q}^{+},(\lambda+\hat{\rho}, \lambda+\hat{\rho})=(\hat{\rho}, \hat{\rho})} a_{\lambda} e^{\lambda} \hat{R}^{-1}
$$

where $a_{\lambda} \in \mathbb{Z}$. This can be rewritten as

$$
\hat{R} e^{\hat{\rho}}=\sum_{\lambda \in \hat{\rho}-\hat{Q}^{+},(\lambda, \lambda)=(\hat{\rho}, \hat{\rho})} a_{\lambda} e^{\lambda}
$$

that is $\operatorname{supp}\left(e^{\hat{\rho}} \hat{R}\right) \subset U$.

Lemma 3.2. The support of $\mathcal{F}_{T^{\prime}}\left(e^{\hat{\rho}} R\right)$ is contained in $U$.

Proof. Let us show that for every $t \in T^{\prime}$, the support of $t\left(e^{\hat{\rho}} R\right)$ is contained in $U$. Recall that the denominator identity of finite dimensional Lie superalgebras takes the form

$$
e^{\rho} R=\mathcal{F}_{W^{\#}}\left(\frac{e^{\rho}}{\prod_{\beta \in S}\left(1+e^{-\beta}\right)}\right)
$$

where $S$ is a maximal isotropic set of roots and the set of simple roots of $\mathfrak{g}$ (which determines $\rho$ ) is assumed to contain $S$ (see Section 2.3). Since $\hat{\rho}-\rho$ is $W^{\#}$-invariant, we have

$$
\begin{aligned}
t\left(e^{\hat{\rho}} R\right) & =t \mathcal{F}_{W^{\#}}\left(\frac{e^{\hat{\rho}}}{\prod_{\beta \in S}\left(1+e^{-\beta}\right)}\right) \\
& =\sum_{w \in W^{\#}}(\operatorname{sgn} w) \frac{e^{t w \hat{\rho}}}{\prod_{\beta \in S}\left(1+e^{-t w \beta}\right)} .
\end{aligned}
$$

For each $w \in W^{\#}$, the support of $e^{t w \hat{\rho}} \cdot \prod_{\beta \in S}\left(1+e^{-t w \beta}\right)^{-1}$ is contained in $t w \hat{\rho}+\mathbb{Z}\{t w \beta \mid \beta \in S\}$ (see Remark 2.4). Since $(\hat{\rho}, \beta)=0$ for all $\beta \in S$ and $(\cdot, \cdot)$ is invariant under the action of the Weyl group and $T^{\prime}$, the assertion follows. 


\section{Proof of the Denominator Identity, $h^{\vee} \neq 0$}

We prove Theorem 1.1 for the case $h^{\vee} \neq 0$ in three steps. We follow the proof of G1]. The first step of the proof is to show that the right hand side of the identity is a well defined element of $\mathcal{R}^{\prime}$. For this we use the properties of roots systems described described in Proposition 4.1). The second step is to show that the support of the difference between the two sides of the equation admits at most one maximal element which is $\hat{\rho}$. The third step of the proof is to show that the coefficient of $e^{\hat{\rho}}$ in both sides is 1 .

4.1. Another form of the denominator identity. We first rewrite $\mathcal{F}_{T^{\prime}}\left(e^{\hat{\rho}} R\right)$ using the denominator identity for finite dimensional Lie superalgebras and use this form to prove Theorem 1.1

Recall that $\pi$ contains a maximal set of isotropic roots $S$ (see Section 2.3). In the first step of the proof we show that $\mathcal{F}_{\hat{W}^{\prime}}\left(e^{\hat{\rho}} \prod_{\beta \in S}\left(1+e^{-\beta}\right)^{-1}\right)$ is well defined. Recall that for $\hat{\mathfrak{g}} \neq A(2 k-1,2 l-1)$, $k \geq l+1, \hat{W}^{\prime}=T^{\prime} \rtimes W^{\prime}$. Then, using the denominator identity for basic Lie superalgebras (2.3), we have that

$$
\begin{aligned}
\mathcal{F}_{T^{\prime}}\left(e^{\hat{\rho}} R\right) & =\mathcal{F}_{T^{\prime}}\left(e^{h^{\vee} \Lambda_{0}} e^{\rho} R\right) \\
& =\mathcal{F}_{T^{\prime}}\left(e^{h^{\vee} \Lambda_{0}} \cdot \mathcal{F}_{W^{\prime}}\left(\frac{e^{\rho}}{\prod_{\beta \in S}\left(1+e^{-\beta}\right)}\right)\right) \\
& =\mathcal{F}_{T^{\prime}}\left(\mathcal{F}_{W^{\prime}}\left(\frac{e^{h^{\vee} \Lambda_{0}+\rho}}{\prod_{\beta \in S}\left(1+e^{-\beta}\right)}\right)\right) \\
& =\mathcal{F}_{\hat{W}^{\prime}}\left(\frac{e^{\hat{\rho}}}{\prod_{\beta \in S}\left(1+e^{-\beta}\right)}\right) .
\end{aligned}
$$

For $\hat{\mathfrak{g}}=A(2 k-1,2 l-1), k \geq l+1, \hat{W}^{\prime} \not \supset T^{\prime}=\operatorname{span}_{\mathbb{Z}}\left\{\varepsilon_{1}, \ldots, \varepsilon_{k}\right\}$ (see Section 2.3). We have that $\hat{W}^{\prime}$ and $W^{\prime}$ are subgroups of index 2 in $\hat{W}_{C_{k}}$ and $W_{C_{k}}$, respectively. Recall that $\hat{W}_{C_{k}}=T^{\prime} \rtimes W_{C_{k}}$ and the sign function is extended from $\hat{W}^{\prime}$ to $\hat{W}_{C_{k}}$ such that $\operatorname{sgn} s_{\varepsilon_{k}}=1$. Note that $S$ and $\hat{\rho}$ are 
$s_{\varepsilon_{k}}$-invariant and hence

$$
\begin{aligned}
\mathcal{F}_{T^{\prime}}\left(e^{\hat{\rho}} R\right) & =\mathcal{F}_{T^{\prime}}\left(e^{h^{\vee} \Lambda_{0}} \cdot \mathcal{F}_{W^{\prime}}\left(\frac{e^{\rho}}{\prod_{\beta \in S}\left(1+e^{-\beta}\right)}\right)\right) \\
& =\mathcal{F}_{T^{\prime}}\left(e^{h^{\vee} \Lambda_{0}} \cdot \mathcal{F}_{W^{\prime}}\left(\frac{1}{2}\left(\frac{e^{\rho}}{\prod_{\beta \in S}\left(1+e^{-\beta}\right)}+s_{2 \varepsilon_{i}} \frac{e^{\rho}}{\prod_{\beta \in S}\left(1+e^{-\beta}\right)}\right)\right)\right) \\
& =\frac{1}{2} \mathcal{F}_{T^{\prime}}\left(e^{h^{\vee} \Lambda_{0}} \cdot \mathcal{F}_{W_{C_{k}}}\left(\frac{e^{\rho}}{\prod_{\beta \in S}\left(1+e^{-\beta}\right)}\right)\right) \\
& =\frac{1}{2} \mathcal{F}_{\hat{W}_{C_{k}}}\left(\frac{e^{\hat{\rho}}}{\prod_{\beta \in S}\left(1+e^{-\beta}\right)}\right) \\
& =\frac{1}{2} \mathcal{F}_{\hat{W}^{\prime}}\left(\frac{e^{\hat{\rho}}}{\prod_{\beta \in S}\left(1+e^{-\beta}\right)}+s_{2 \varepsilon_{i}} \frac{e^{\hat{\rho}}}{\prod_{\beta \in S}\left(1+e^{-\beta}\right)}\right) \\
& =\mathcal{F}_{\hat{W}^{\prime}}\left(\frac{e^{\hat{\rho}}}{\prod_{\beta \in S}\left(1+e^{-\beta}\right)}\right)
\end{aligned}
$$

We get that the denominator identity can be written as

$$
e^{\hat{\rho}} \hat{R}=\mathcal{F}_{\hat{W}^{\prime}}\left(\frac{e^{\hat{\rho}}}{\prod_{\beta \in S}\left(1+e^{-\beta}\right)}\right) .
$$

The fact that $\mathcal{F}_{\hat{W}^{\prime}}\left(e^{\hat{\rho}} \prod_{\beta \in S}\left(1+e^{-\beta}\right)^{-1}\right)$ is well defined implies that so is $\mathcal{F}_{T^{\prime}}\left(e^{\hat{\rho}} R\right)$. However, the converse implication does not hold since it is not necessarily possible to open the parenthesis in the third equality of (4.1) and the fourth equality of (4.2). In fact, neither $\mathcal{F}_{\hat{W}^{\prime}}\left(e^{\hat{\rho}} \prod_{\beta \in S}\left(1+e^{-\beta}\right)^{-1}\right)$ nor $\mathcal{F}_{\hat{W}^{\prime \prime}}\left(e^{\hat{\rho}} \prod_{\beta \in S}\left(1+e^{-\beta}\right)^{-1}\right)$ is well defined for $\hat{\mathfrak{g}}=A(2 k-1,2 k-1)^{(2)}$.

4.2. Choice of simple roots. For the rest of this section we will assume that our choice of simple roots satisfies the conditions of the following proposition. The proof of existence of such choices is given in Appendix 6.2 .

Proposition 4.1. For every twisted affine Lie superalgebra with $h^{\vee} \neq 0$, there exists a choice of set of simple roots $\hat{\pi}$ such that

(i) for all $\alpha \in \hat{\pi},(\alpha, \alpha) \geq 0$;

(ii) for $\hat{\mathfrak{g}} \neq A(2 k, 2 k+1)^{(2)}, A(2 k, 2 k-1)^{(2)}$ and $G(3)^{(2)}$, one has $\left(\alpha_{0}, \alpha_{0}\right)>0$.

Remark 4.2. Suppose that the set of simple roots satisfies (i), then $\left(\hat{\rho}, \hat{Q}^{+}\right) \geq 0$.

Suppose that we fix the "finite part" $\mathfrak{g}$ in $\hat{\mathfrak{g}}$, then $e^{\hat{\rho}} \hat{R}$ and $e^{\hat{\rho}} R$ are independent of the choice of simple roots. Thus, it suffices to prove Theorem 1.1 for a choice satisfying the conditions of Proposition 4.1. However, the choice of the finite part is not unique. For example, the Lie algebra $B_{l}^{(1)}$ has two non-isomorphic maximal finite subalgebras, namely $B_{l}$ and $D_{l}$ each obtained by removing one vertex from the Dynkin diagram of $B_{l}^{(1)}$. The situation is more complicated for Lie superalgebras since the Dynkin diagram is not unique and one can obtain different finite parts by removing a vertex in different diagrams 
The denominator identity for non-twisted Lie superalgebras ([G1, GR]) also depends on the choice of the finite part. However, for twisted algebras this problem is more significant since the choice of the finite part is not canonical. Consider for example the first set of simple roots described in Table 2. One can see that if we take the finite part that corresponds to the Dynkin diagram of $\hat{\pi} \backslash\left\{\varepsilon_{k}\right\}$, the conditions of Proposition 4.1 still hold and hence our proof for Theorem 1.1 applies. However, it is not clear whether one can also do so for other choices of simple roots. It is interesting to look for a proof of the denominator identity (twisted and not-twisted) that is independent of the choice of simple roots and hence will apply to all finite parts.

4.3. Step I. Let us show that $\mathcal{F}_{\hat{W}^{\prime}}\left(e^{\hat{\rho}} R\right)$ is well defined. By Section 4.1. $\mathcal{F}_{T^{\prime}}\left(e^{\hat{\rho}} R\right)$ is well-defined as well.

Proposition 4.3. The formal sum $\mathcal{F}_{\hat{W}^{\prime}}\left(\frac{e^{\hat{\rho}}}{\prod_{\beta \in S}\left(1+e^{-\beta}\right)}\right)$ is in $\mathcal{R}^{\prime}$ and its support lies in $\hat{\rho}-\hat{Q}^{+}$.

Proof. We extend the proof in [G1, 2.4.1] to twisted affine Lie superalgebras. By [HS, 5] and [S, 4.10] the set of principal roots of $\hat{\Delta}$ is equal to the set of simple roots of $\hat{\Delta}_{\overline{0}}^{+}$. Hence $\hat{W}^{\prime}$ is a subgroup of the group $W_{+}$introduced in Lemma 2.1 and $\Pi_{+} \subseteq \hat{\pi}^{\prime}$. By Remark 2.4 for every $w \in \hat{W}^{\prime}$, the maximal element of the support $w\left(e^{\hat{\rho}} \cdot \prod_{\beta \in S}\left(1+e^{-\beta}\right)^{-1}\right)$ is $w \hat{\rho}+\sum_{\beta \in S: w \beta \in \hat{\Delta}^{-}} w \beta$. By Lemma 2.1.(i), $w \hat{\rho} \leq \hat{\rho}$. Hence

$$
\operatorname{supp}\left(w \frac{e^{\hat{\rho}}}{\prod_{\beta \in S}\left(1+e^{-\beta}\right)}\right) \subset w \hat{\rho}-\hat{Q}^{+} \subset \hat{\rho}-\hat{Q}^{+} .
$$

So it suffices to show that the set $G_{r}:=\left\{w \in \hat{W}^{\prime} \mid\right.$ ht $\left.\left(\hat{\rho}-w \hat{\rho}+\sum_{\beta \in S: w \beta \in \hat{\Delta}^{-}} w \beta\right) \leq r\right\}$ is finite for every $r$. This set is contained in the set $H_{r}:=\left\{w \in \hat{W}^{\prime} \mid\right.$ ht $\left.(\hat{\rho}-w \hat{\rho}) \leq r\right\}$. We will show that $H_{r}$ is finite. By Lemma 2.1. (ii) every element in $H_{r}$ is of the form $w_{0} s_{i_{1}} w_{1} \cdot \ldots \cdot s_{i_{j}} w_{j}$ where $w_{i} \in H_{0}, j \leq r$ and $s_{i_{j}}$ are simple reflections for all $i$.

Let us show that $H_{0}$ is a finite subgroup of $\hat{W}^{\prime}$. By Lemma 2.1.(iii) $H_{0}$ is the subgroup generated by $\left\{s_{\alpha} \mid \alpha \in \Pi_{+}\right.$and $\left.\left\langle\hat{\rho}, \alpha^{\vee}\right\rangle=0\right\}$. Let $\Sigma$ and $\Sigma_{0}$ be the Dynkin diagrams of $\hat{\Delta}^{\prime}$ and $\left\{\alpha \in \Pi_{+} \mid\left\langle\hat{\rho}, \alpha^{\vee}\right\rangle=0\right\}$, respectively. The inclusion $\Sigma_{0} \subset \Sigma$ is proper. Indeed, since $h^{\vee} \neq 0$, there exists a simple root $\alpha$ such that $\frac{1}{2}(\alpha, \alpha)=(\hat{\rho}, \alpha) \neq 0$. Since $(\alpha, \alpha) \neq 0$, either $\alpha$ or $2 \alpha$ is a principal root. By Proposition 4.1.(i), $(\alpha, \alpha)>0$. Thus, either $\alpha$ or $2 \alpha$ belongs to $\hat{\Delta}^{\prime}$, so $\left(\hat{\rho}, \hat{\Delta}^{\prime}\right) \neq 0$ and hence $\Sigma_{0} \neq \Sigma$. Since $\Sigma$ is affine and indecomposable and $\Sigma_{0}$ is a proper subdiagram of $\Sigma$, we get that $\Sigma_{0}$ is of finite type and thus $H_{0}$ is finite as asserted.

4.4. Step II. The next step of the proof is to show that the support of the difference between the two sides of (1.1) admits at most one maximal element which is $\hat{\rho}$. We do this by showing that $\hat{\rho}$ is the only element in $U$ which is a maximal element of a regular $\hat{W}^{\prime}$-orbit. We multiply $e^{\hat{\rho}} \hat{R}$ and $\mathcal{F}_{T^{\prime}}\left(e^{\hat{\rho}} R\right)$ by $e^{\hat{\rho}^{\prime}-\hat{\rho}} \hat{R}_{1}$ so that they will belong to the algebra $\mathcal{R}_{\hat{W}^{\prime}}$ where the action of $\hat{W}^{\prime}$ can be applied to the support of a series (that is lemmas 2.5] and 2.6 are applicable).

Proposition 4.4. If $Y:=e^{\hat{\rho}} \hat{R}-\mathcal{F}_{T^{\prime}}\left(e^{\hat{\rho}} R\right)$ is non zero, the only maximal element in the support of $Y$ is $\hat{\rho}$.

To prove this proposition, we use the following lemma about affine Lie algebras: 
Lemma 4.5. (G1, 3.1.1]) Let $\mathfrak{a}$ be an affine Lie algebra with set of simple roots $\pi_{\mathfrak{a}}$, and $W_{\mathfrak{a}}$ its Weyl group. Let $\rho_{\mathfrak{a}}$ be such that $\left(\rho_{\mathfrak{a}}, \alpha\right)=\frac{1}{2}(\alpha, \alpha)$ for all $\alpha \in \pi_{\mathfrak{a}}$. Suppose $\lambda \in \sum_{\alpha \in \pi_{\mathfrak{a}}} \mathbb{Q} \alpha$ is such that $\lambda+\rho_{\mathfrak{a}}$ is a maximal element in a regular $W_{\mathfrak{a}}$-orbit and $\left\langle\lambda, \alpha^{\vee}\right\rangle \in \mathbb{Z}$ for all $\alpha \in \pi_{\mathfrak{a}}$. Then $\lambda \in \mathbb{Q} \delta$ where $\delta$ is the minimal imaginary root of $\mathfrak{a}$.

Proof of Proposition 4.4. Let $\mu$ be a maximal element in $\operatorname{supp} Y$. Let us show that $\mu=\hat{\rho}$. By Section $3(\mu, \mu)=(\hat{\rho}, \hat{\rho})$. The element $\mu+\hat{\rho}^{\prime}-\hat{\rho}$ is a maximal element of the support of $e^{\hat{\rho}^{\prime}-\hat{\rho}} \hat{R}_{1} \cdot Y$. By the $\hat{W}^{\prime}$-invariance of $e^{\hat{\rho}^{\prime}-\hat{\rho}} \hat{R}_{1}$ (see Remark 2.7) and (4.1), (4.2), we have the following equality

$$
e^{\hat{\rho}^{\prime}-\hat{\rho}} \hat{R}_{1} \cdot Y=e^{\hat{\rho}^{\prime}} \hat{R}_{0}-\mathcal{F}_{\hat{W}^{\prime}}\left(\frac{e^{\hat{\rho}^{\prime}} \hat{R}_{1}}{\prod_{\beta \in S}\left(1+e^{-\beta}\right)}\right)
$$

Note that the summands in the right hand side of (4.3) are $\hat{W}^{\prime}$-anti-invariant elements of $\mathcal{R}_{\hat{W}^{\prime}}$ (using Lemma 2.5). By Lemma [2.6] the support of $e^{\hat{\rho}^{\prime}-\hat{\rho}} \hat{R}_{1} \cdot Y$ is a union of regular orbits. For an element in $\lambda=\mathbb{Q} \hat{\pi}$, we write $\lambda=p^{\prime}(\lambda)+p^{\prime \prime}(\lambda)$ where $p^{\prime}(\lambda) \in \mathbb{Q} \hat{\pi}^{\prime}$ and $p^{\prime \prime}(\lambda) \in \mathbb{Q} \pi^{\prime \prime}$. One has that $p^{\prime}\left(\mu+\hat{\rho}^{\prime}-\hat{\rho}\right)=p^{\prime}(\mu-\hat{\rho})+\hat{\rho}^{\prime}$ is a maximal element in its $\hat{W}^{\prime}$-orbit. By Lemma 4.5. $p^{\prime}(\mu-\hat{\rho})=-s \delta, s \in \mathbb{Q}$. Recall that

$$
\begin{aligned}
(\hat{\rho}, \hat{\rho}) & =(\mu, \mu) \\
& =\left(\hat{\rho}+p^{\prime}(\mu-\hat{\rho})+p^{\prime \prime}(\mu-\hat{\rho}), \hat{\rho}+p^{\prime}(\mu-\hat{\rho})+p^{\prime \prime}(\mu-\hat{\rho})\right) \\
& =\left(\hat{\rho}-s \delta+p^{\prime \prime}(\mu-\hat{\rho}), \hat{\rho}-s \delta+p^{\prime \prime}(\mu-\hat{\rho})\right)
\end{aligned}
$$

which implies that

$$
\left(p^{\prime \prime}(\mu-\hat{\rho}), p^{\prime \prime}(\mu-\hat{\rho})\right)+2\left(\hat{\rho},-s \delta+p^{\prime \prime}(\mu-\hat{\rho})\right)=0 .
$$

One has $\hat{\rho}-s \delta+p^{\prime \prime}(\mu-\hat{\rho}) \in \operatorname{supp} Y$ and by Lemma 4.3, $\operatorname{supp} Y \subset \hat{\rho}-\hat{Q}^{+}$. Hence $\left(\hat{\rho},-s \delta+p^{\prime \prime}(\mu-\hat{\rho})\right) \leq$ 0 by Remark 4.2 Since $(\cdot, \cdot)$ is negative definite on $\Delta^{\prime \prime},\left(p^{\prime \prime}(\mu-\hat{\rho}), p^{\prime \prime}(\mu-\hat{\rho})\right) \leq 0$ and we get that $p^{\prime \prime}(\mu-\hat{\rho})=0$ and hence $s=0$. Thus, $\mu=\hat{\rho}$ and the assertion follows.

Remark 4.6. When $h^{\vee}=0$, there are algebras for which there is no choice a set of simple roots such that $\left(\hat{\rho}, \hat{Q}^{+}\right) \geq 0$ and hence we can not use the argument described in this proof.

4.5. Step III. Let us complete the proof of Theorem 1.1 for $h^{\vee} \neq 0$ by showing that the coefficients of $e^{\hat{\rho}}$ are equal on both sides of the equation. That is, we show that $\hat{\rho}$ does not belong to the support of $e^{\hat{\rho}} \hat{R}-\mathcal{F}_{T^{\prime}}\left(e^{\hat{\rho}} R\right)$.

Clearly the coefficient of $e^{\hat{\rho}}$ in $e^{\hat{\rho}} \hat{R}$ is 1 . On the other hand, we have:

Proposition 4.7. The coefficient of $e^{\hat{\rho}}$ in $\mathcal{F}_{\hat{W}^{\prime}}\left(\frac{e^{\hat{\rho}}}{\prod_{\beta \in S}\left(1+e^{-\beta}\right)}\right)$ is 1 .

Proof. Note that the coefficient of $e^{\hat{\rho}}$ in $\sum_{w \in \hat{W}^{\prime}}(\operatorname{sgn} w) w\left(\frac{e^{\hat{\rho}}}{\prod_{\beta \in S}\left(1+e^{-\beta}\right)}\right)$ is equal to $\sum_{w \in A}(\operatorname{sgn} w)$ where $A:=\left\{w \in \hat{W}^{\prime} \mid w \hat{\rho}=\hat{\rho}, w \beta \in \hat{\Delta}^{+}\right.$for all $\left.\beta \in S\right\}$ (see Remark 2.4). We prove the proposition by showing that $A=\{1\}$.

Case 1: $\left(\alpha_{0}, \alpha_{0}\right)>0$. We show that the stabilizer of $\hat{\rho}$ in $\hat{W}^{\prime}$ is trivial. Similarly to the argument of Proposition 4.3, Lemma 2.1 (iii) yields that the stabilizer of $\hat{\rho}$ in $\hat{W}^{\prime}$ is generated by reflections with respect to roots in $\hat{\pi}^{\prime}$. By Proposition 4.1.(i), $\alpha_{0} \in \hat{\pi}^{\prime}$ but $s_{\alpha_{0}}$ is not in the stabilizer since $\left(\alpha_{0}, \alpha_{0}\right)=\frac{1}{2}\left(\alpha_{0}, \hat{\rho}\right) \neq 0$. Hence the stabilizer is generated by reflections with respect to roots in 
$\pi^{\prime}$ so $\operatorname{Stab}_{\hat{W}}, \hat{\rho}$ lies in $W^{\prime}$ and thus coincides with $\operatorname{Stab}_{W^{\prime}} \rho$. We get that the coefficient of $e^{\hat{\rho}}$ in $\mathcal{F}_{\hat{W}^{\prime}}\left(\frac{e^{\hat{\rho}}}{\prod_{\beta \in S}\left(1+e^{-\beta}\right)}\right)$ is the same as in $\mathcal{F}_{W^{\prime}}\left(\frac{e^{\hat{\rho}}}{\prod_{\beta \in S}\left(1+e^{-\beta}\right)}\right)$. Since $\hat{\rho}-\rho$ is $W^{\prime}$-invariant, we get that it is equal to the coefficient of $e^{\rho}$ in $\mathcal{F}_{W^{\prime}}\left(\frac{e^{\rho}}{\prod_{\beta \in S}\left(1+e^{-\beta}\right)}\right)$. By the denominator identity for finite dimensional Lie superalgebras it is equal to the coefficient of $e^{\rho}$ in $e^{\rho} R$ which is clearly 1 .

Case 2: $G(3)^{(2)}$. In this case $S=\left\{\varepsilon_{3}-\varepsilon_{2}-\varepsilon_{1}\right\}, \hat{\Delta}^{\prime}=\hat{\Delta}_{\overline{0}} \backslash\left\{2 s \delta \pm 2 \varepsilon_{3}\right\}_{s \in \mathbb{Z}}$ and $\hat{\rho}=3 \Lambda_{0}-$ $\varepsilon_{3}+\varepsilon_{1}+\varepsilon_{2}$. By Lemma 2.1.(iii), the stabilizer of $\hat{\rho}$ is generated by reflections with respect to the principal roots. The principal roots are $\left\{2 \varepsilon_{1}, 2 \varepsilon_{2}, \delta-3 \varepsilon_{2}-\varepsilon_{1}\right\}$ and so $\operatorname{Stab}_{\hat{W}^{\prime}} \hat{\rho}=\left\{1, s_{\delta-3 \varepsilon_{2}-\varepsilon_{1}}\right\}$. Since $s_{\delta-3 \varepsilon_{2}-\varepsilon_{1}}\left(\varepsilon_{3}-\varepsilon_{2}-\varepsilon_{1}\right) \notin \hat{\Delta}^{+}, A=\{1\}$.

Case 3: $A(2 k, 2 k+1)^{(2)}$. In this case $\hat{\rho}=\Lambda_{0}+\frac{1}{2}\left(\sum_{i=1}^{k}\left(\varepsilon_{i}-\delta_{i}\right)+\varepsilon_{k+1}\right)$ and we take $S=$ $\left\{\delta_{i}-\varepsilon_{i+1}\right\}_{i=1, \ldots, k}$. Here $\hat{\Delta}^{\prime}=\left\{s \delta_{s \neq 0}, s \delta \pm \varepsilon_{g} \pm \varepsilon_{h}, 2 s \delta \pm 2 \varepsilon_{h}\right\}$ and $T^{\prime}=\left\{t_{\mu} \mid \mu \in M\right\}$ where $M=$ $\operatorname{span}_{\mathbb{Z}}\left\{ \pm \varepsilon_{g} \pm \varepsilon_{h}\right\}, s \in \mathbb{Z}, g \neq h$ and $1 \leq g, h \leq k+1$.

Let $w \in A$. We show that $w=1$. Write $w=t_{\mu} y$ where $y \in W^{\prime}$ and $\mu \in \operatorname{span}_{\mathbb{Z}}\left\{ \pm \varepsilon_{g} \pm \varepsilon_{h}\right\}$. Then $w S \subset \hat{\Delta}^{+}$means that $w\left(\delta_{i}-\varepsilon_{i+1}\right)=\delta_{i}-y \varepsilon_{i+1}+\left(\mu, y \varepsilon_{i+1}\right) \delta \in \hat{\Delta}^{+}$. Hence $\left(\mu, y \varepsilon_{i}\right) \geq 0$ for all $i=2, \ldots, k+1$. On the other hand, $\hat{\rho}=w \hat{\rho}$ means that

$$
\begin{aligned}
\hat{\rho} & =y \hat{\rho}+h^{\vee} \mu-\left(\left(\hat{\rho}, y^{-1} \mu\right)+\frac{(\mu, \mu)}{2} h^{\vee}\right) \delta \\
& =y \hat{\rho}+\mu-\frac{1}{2}\left(\left(\varepsilon_{1}+\ldots+\varepsilon_{k+1}, y^{-1} \mu\right)+\left(y^{-1} \mu, y^{-1} \mu\right)\right) \delta .
\end{aligned}
$$

Write $y^{-1} \mu=\sum a_{i} \varepsilon_{i}$ and $\sum a_{i}=0(\bmod 2)$. Then

$$
\begin{aligned}
0 & =\left(\varepsilon_{1}+\ldots+\varepsilon_{k+1}, y^{-1} \mu\right)+\left(y^{-1} \mu, y^{-1} \mu\right) \\
& =\sum a_{i}\left(a_{i}+1\right) .
\end{aligned}
$$

Since $a_{i} \in \mathbb{Z}$, we get that $a_{i} \in\{0,-1\}$. Since $a_{i}=\left(\mu, y \varepsilon_{i}\right) \geq 0$ for $i=2, \ldots, k+1$, we have $a_{2}, \ldots, a_{k+1}=0$ and hence $a_{1}=0$. Hence $\mu=0$ and $w=y \in W^{\prime}$. Since $w \hat{\rho}=\hat{\rho}$, we get that $w$ permutes $\varepsilon_{1} \ldots, \varepsilon_{k+1}$ (no sign change). Note that $\delta_{i}-\varepsilon_{j} \in \Delta^{-}$if $j \leq i$ and so the only permutation $w$ such that $w S \subset \Delta^{+}$is 1 . Thus, $A=\{1\}$.

Case 4: $A(2 k, 2 k-1)^{(2)}$. In this case $S=\left\{\delta_{i}-\varepsilon_{i}\right\}_{i=1, \ldots, k}$ and $\hat{\rho}=\Lambda_{0}+\frac{1}{2} \sum_{i=1}^{k}\left(\varepsilon_{i}-\delta_{i}\right)$. Here $\hat{\Delta}^{\prime}=\left\{s \delta_{s \neq 0}, s \delta \pm \varepsilon_{g} \pm \varepsilon_{h}, s \delta \pm \varepsilon_{g},(2 s+1) \delta \pm 2 \varepsilon_{g}\right\}$ where $s \in \mathbb{Z}, g \neq h$ and $1 \leq g, h \leq k$. Let $w \in A$. We show that $w=1$. Write $w=t_{\mu} y$ where $y \in W^{\prime}$ and $\mu \in \operatorname{span}_{\mathbb{Z}}\left\{\varepsilon_{1}, \ldots, \varepsilon_{k}\right\}$. Then $w S \subset \hat{\Delta}^{+}$means that $w\left(\delta_{i}-\varepsilon_{i}\right)=\delta_{i}-y \varepsilon_{i}+\left(\mu, y \varepsilon_{i}\right) \delta \in \hat{\Delta}^{+}$. Hence $\left(\mu, y \varepsilon_{i}\right) \geq 0$ for all $i=1, \ldots, k$. On the other hand, $\hat{\rho}=w \hat{\rho}$ means that

$$
\begin{aligned}
\hat{\rho} & =w \hat{\rho}=y \hat{\rho}+h^{\vee} \mu-\left(\left(\hat{\rho}, y^{-1} \mu\right)+\frac{(\mu, \mu)}{2} h^{\vee}\right) \delta \\
& =y \hat{\rho}+\mu-\frac{1}{2}\left(\left(\varepsilon_{1}+\ldots+\varepsilon_{k}, y^{-1} \mu\right)+(\mu, \mu)\right) \delta .
\end{aligned}
$$

Since $\left(\varepsilon_{1}+\ldots+\varepsilon_{k}, y^{-1} \mu\right) \geq 0$ and $(\mu, \mu) \geq 0$, we get that $\mu=0$ and so $w=y \in W^{\prime}$. Thus, $w \hat{\rho}=\hat{\rho}$ implies that $w$ permutes $\varepsilon_{1} \ldots, \varepsilon_{k}$ (no sign change). Note that $\delta_{i}-\varepsilon_{j} \in \Delta^{-}$if $j<i$ and hence the only permutation $w$ such that $w S \subset \hat{\Delta}^{+}$is 1 . Thus, $A=\{1\}$. 


\section{Proof of the Denominator Identity, $h^{\vee}=0$}

In this section we prove Theorem 1.1 for the case $h^{\vee}=0$, in three steps. The first step is to show that the sum $\mathcal{F}_{T^{\prime}}\left(R e^{\hat{\rho}}\right)$ is well defined and belongs to $\mathcal{R}$. In the second step, we show that $\hat{R}^{-1} e^{-\hat{\rho}}$. $\mathcal{F}_{T^{\prime}}\left(R e^{\hat{\rho}}\right)$ takes the form $f(q)$. In the third step we compute $f(q)$ using a proper evaluation. The case $h^{\vee}=0$ consists of the algebras $A(2 k-1,2 k-1)^{(2)}, A(2 k, 2 k)^{(4)}$ and $D(k+1, k)^{(2)}$. We first describe the even roots and the translation groups.

5.1. Description of the root system and the Weyl group. We describe the set of even roots $\Delta_{\overline{0}}=\Delta^{\prime} \sqcup \Delta^{\prime \prime}$ of $\mathfrak{g}$, and the translation groups $T^{\prime}$ and $T^{\prime \prime}$ of the Weyl group. We denote by $\mathbb{C}\left[t^{m}, t^{-m}\right](\hat{\mathfrak{k}})$, the affine Lie algebra which is isomorphic to $\hat{\mathfrak{k}}$ where $t \otimes g \in \hat{\mathfrak{k}}, g \in \mathfrak{k}$ is mapped to $t^{m} \otimes g \in \mathbb{C}\left[t^{m}, t^{-m}\right](\hat{\mathfrak{k}})$.

5.1.1. $A(2 k-1,2 k-1)^{(2)}$. In this case $\mathfrak{g}=D(k, k)$. The set of even roots of $\mathfrak{g}$ is $\Delta^{\prime} \sqcup \Delta^{\prime \prime}$,

$$
\Delta^{\prime}=\left\{\delta_{i} \pm \delta_{j} \mid i \neq j\right\}, \quad \Delta^{\prime \prime}=\left\{\varepsilon_{i} \pm \varepsilon_{j} \mid i \neq j\right\} \cup\left\{2 \varepsilon_{i}\right\}
$$

where $i, j=1, \ldots, k$. The set of even roots of $\hat{\mathfrak{g}}$ is $\hat{\Delta}_{\overline{0}}=\hat{\Delta}^{\prime} \cup \hat{\Delta}^{\prime \prime}$ where $\hat{\Delta}^{\prime}$ and $\hat{\Delta}^{\prime \prime}$ are the root system of $A_{2 k-1}^{(2)}$. The translation subgroups are $T^{\prime}=\left\{t_{\mu} \mid \mu \in M^{\prime}\right\}$ where $M^{\prime}=\operatorname{span}_{\mathbb{Z}}\left\{\delta_{1}, \ldots, \delta_{k}\right\}$ and $T^{\prime \prime}=\left\{t_{\mu} \mid \mu \in M^{\prime \prime}\right\}$ where $M^{\prime \prime}=\operatorname{span}_{\mathbb{Z}}\left\{\varepsilon_{i} \pm \varepsilon_{j}\right\}$.

Recall that $\hat{W}^{\prime} \nsupseteq T^{\prime}$ and we embed $W^{\prime}$ and $\hat{W}^{\prime}$ in $W_{C_{k}}=\left\langle W^{\prime}, s_{\delta_{k}}\right\rangle$ and $\hat{W}_{C_{k}}=\left\langle\hat{W}^{\prime}, s_{\delta_{k}}\right\rangle$, respectively. One has $\hat{W}_{C_{k}}=T^{\prime} \rtimes W_{C_{k}}$.

5.1.2. $A(2 k, 2 k)^{(4)}$. In this case $\mathfrak{g}=B(k, k)$. The set of even roots of $\mathfrak{g}$ is $\Delta^{\prime} \sqcup \Delta^{\prime \prime}$,

$$
\Delta^{\prime}=\left\{\delta_{i} \pm \delta_{j} \mid i \neq j\right\} \cup\left\{\delta_{i}\right\}, \quad \Delta^{\prime \prime}=\left\{\varepsilon_{i} \pm \varepsilon_{j} \mid i \neq j\right\} \cup\left\{2 \varepsilon_{i}\right\}
$$

where $i, j=1, \ldots, k$. The set of even roots of $\hat{\mathfrak{g}}$ is $\hat{\Delta}_{\overline{0}}=\hat{\Delta}^{\prime} \cup \hat{\Delta}^{\prime \prime}$ where $\hat{\Delta}^{\prime}$ and $\hat{\Delta}^{\prime \prime}$ are the root systems of $\mathbb{C}\left[t^{4}, t^{-4}\right]\left(A_{2 k}^{(2)}\right)$ and $\mathbb{C}\left[t^{2}, t^{-2}\right]\left(A_{2 k}^{(2)}\right)$, respectively. The translation subgroups are $T^{\prime}=\left\{t_{\mu} \mid \mu \in M^{\prime}\right\}$ where $M^{\prime}=\operatorname{span}_{\mathbb{Z}}\left\{2 \delta_{1}, \ldots, 2 \delta_{k}\right\}$ and $T^{\prime \prime}=\left\{t_{\mu} \mid \mu \in M^{\prime \prime}\right\}$ where $M^{\prime \prime}=$ $\operatorname{span}_{\mathbb{Z}}\left\{2 \varepsilon_{1}, \ldots, 2 \varepsilon_{k}\right\}$. As we shall see, in this case it is possible to swap between $\hat{\Delta}^{\prime}$ and $\hat{\Delta}^{\prime \prime}$ and the proof works.

5.1.3. $D(k+1, k)^{(2)}$. In this case $\mathfrak{g}=B(k, k)$ as well, and the set of even roots of $\mathfrak{g}$ is the same as in 5.1.2. The set of even roots of $\hat{\mathfrak{g}}$ is $\hat{\Delta}_{\overline{0}}=\hat{\Delta}^{\prime} \cup \hat{\Delta}^{\prime \prime}$ where $\hat{\Delta}^{\prime}$ and $\hat{\Delta}^{\prime \prime}$ are the root systems of $D_{k+1}^{(2)}$ and $\mathbb{C}\left[t^{2}, t^{-2}\right]\left(C_{k}^{(1)}\right)$, respectively. The translation subgroups are $T^{\prime}=\left\{t_{\mu} \mid \mu \in M^{\prime}\right\}$ where $M^{\prime}=\operatorname{span}_{\mathbb{Z}}\left\{2 \delta_{1}, \ldots, 2 \delta_{k}\right\}$ and $T^{\prime \prime}=\left\{t_{\mu} \mid \mu \in M^{\prime \prime}\right\}$ where $M^{\prime \prime}=\operatorname{span}_{\mathbb{Z}}\left\{2 \varepsilon_{1}, \ldots, 2 \varepsilon_{k}\right\}$. In this case, one can swap between $\hat{\Delta}^{\prime}$ and $\hat{\Delta}^{\prime \prime}$. As we shall see, in this case it is possible to swap between $\hat{\Delta}^{\prime}$ and $\hat{\Delta}^{\prime \prime}$ and the proof works.

5.2. Step I. Let us show that $\mathcal{F}_{T^{\prime}}\left(e^{\rho} R\right)$ is a well defined element of $\mathcal{R}$. In the case $\hat{\mathfrak{g}}=A(2 k-1,2 k-1)^{(2)}$, we use a method from [GR, 2.1] and in the cases $A(2 k, 2 k)^{(4)}$ and $D(k+1, k)^{(2)}$, we use the denominator identity for $B(k, k)$.

Note that when $h^{\vee}=0, \hat{\rho}=\rho$.

Lemma 5.1. For $\hat{\mathfrak{g}}=A(2 k-1,2 k-1)^{(2)}, \mathcal{F}_{T^{\prime}}\left(e^{\rho} R\right)$ is well defined and belongs to the algebra $\mathcal{R}$. 
Proof. Let us show that for every $w \in T^{\prime}$, the maximal element of $\operatorname{supp} w\left(e^{\rho} R\right)$ is less than $\rho+$ $\sum_{\beta \in \Delta_{1}^{+}} \beta$ and that for every $\nu \leq \rho+\sum_{\beta \in \Delta_{1}^{+}} \beta$, there are only finitely many $w \in T^{\prime}$ such that the maximal element of $\operatorname{supp} w\left(e^{\rho} R\right)$ is larger than $\nu$. The latter implies that $\mathcal{F}_{T^{\prime}}\left(e^{\rho} R\right)$ is well defined, whereas the former implies that

$$
\operatorname{supp}\left(\mathcal{F}_{T^{\prime}}\left(e^{\rho} R\right)\right) \subset \rho+\sum_{\beta \in \Delta_{1}^{+}} \beta-\hat{Q}^{+},
$$

that is $\mathcal{F}_{T^{\prime}}\left(e^{\rho} R\right)$ belongs to $\mathcal{R}$.

One has

$$
\max \operatorname{supp} w\left(e^{\rho} R\right)=w \rho-\sum_{\alpha \in \Delta_{0}^{+}: w \alpha<0} w \alpha+\sum_{\alpha \in \Delta_{1}^{+}: w \alpha<0} w \alpha .
$$

Each $w \in T^{\prime}$ is of the form $w=t_{\mu}$ where $\mu \in \sum_{i=1}^{n} \mathbb{Z} \delta_{i}$. Note that for every $\beta \in \mathbb{Q} \pi, w \beta<0$ if and only if $(\beta, \mu)>0$. We obtain that

$$
\max \operatorname{supp} t_{\mu}\left(e^{\rho} R\right)=-v(\mu)+(v(\mu), \mu) \delta,
$$

where

We show that

$$
v(\mu)=-\rho+\sum_{\alpha \in \Delta_{0}^{+}: t_{\mu}(\alpha)<0} \alpha-\sum_{\alpha \in \Delta_{1}^{+}: t_{\mu}(\alpha)<0} \alpha .
$$

(i) for every $\mu$ such that $t_{\mu} \in T^{\prime}, \quad(v(\mu), \mu) \leq 0$;

(ii) for every $N>0,\{\mu \mid(v(\mu), \mu) \geq-N\}$ is a finite set.

By (5.1), we see that condition (ii) insures that only finitely many maximal elements can apear above a certain weight and condition (i) means that for all $\mu$ one has

$$
\max \operatorname{supp}\left(t_{\mu}\left(e^{\rho} R\right)\right) \leq-v(\mu) \leq \rho+\sum_{\beta \in \Delta_{1}^{+}} \beta .
$$

Let us verify (i) and (ii). Recall that $\mu$ has the form $\mu=\sum_{i=1}^{k} n_{i} \delta_{i}$, where $n_{i} \in \mathbb{Z}$. Write $v(\mu)=v^{\prime}+v^{\prime \prime}$, where $v^{\prime}=\sum_{i=1}^{k} a_{i} \delta_{i}$ and $v^{\prime \prime}$ lies in the span of the $\varepsilon_{i}$-s. Let us show that if $n_{i}>0$ then $a_{i} \leq-\frac{1}{2}$ and if $n_{i}<0$ then $a_{i} \geq \frac{1}{2}$. We shall then have that

$$
(v(\mu), \mu)=\sum a_{i} n_{i} \leq-\frac{1}{2} \sum_{n_{i}>0} n_{i}+\frac{1}{2} \sum_{n_{i}<0} n_{i} \leq 0
$$

and hence the set $\{\mu \mid(v(\mu), \mu) \geq-N\}$ is a subset of $\left\{\sum_{i=1}^{k} n_{i} \delta_{i}\left|\frac{1}{2} \sum\right| n_{i} \mid<N\right\}$ which is finite. One has $\rho=0$ and

$$
\begin{aligned}
& \Delta_{\overline{0}}^{+}=\left\{\delta_{i} \pm \delta_{j} \mid 1 \leq i<j \leq k\right\} \cup\left\{\varepsilon_{i} \pm \varepsilon_{j} \mid 1 \leq i<j \leq k\right\} \cup\left\{2 \varepsilon_{i} \mid 1 \leq i \leq k\right\} \\
& \Delta_{\overline{1}}^{+}=\left\{\delta_{i} \pm \varepsilon_{j} \mid 1 \leq i<j \leq k\right\} \cup\left\{\varepsilon_{i} \pm \delta_{j} \mid 1 \leq i \leq j \leq k\right\} .
\end{aligned}
$$

Hence

$$
\begin{aligned}
& \left\{\alpha \in \Delta_{\overline{0}}^{+} \mid(\alpha, \mu)>0\right\}=\left\{\delta_{i}-\delta_{j} \mid i<j, n_{i}>n_{j}\right\} \cup\left\{\delta_{i}+\delta_{j} \mid i<j, n_{i}+n_{j}>0\right\} \\
& \left\{\alpha \in \Delta_{\overline{1}}^{+} \mid(\alpha, \mu)>0\right\}=\left\{\varepsilon_{i}-\delta_{j} \mid i \leq j, n_{j}<0\right\} \cup\left\{\delta_{i}-\varepsilon_{j} \mid i<j, n_{i}>0\right\} \cup\left\{\delta_{i}+\varepsilon_{j} \mid n_{i}>0\right\},
\end{aligned}
$$

where $1 \leq i, j \leq k$. So for $n_{i}>0$, one has $a_{i} \leq(2 k-i-1)-(2 k-i)=-1$ and for $n_{i}<0$, one has $a_{i} \geq-(i-1)+i=1$ as required. 
Remark 5.2. Note that the above argument does not apply if one would take $T^{\prime \prime}$ instead of $T^{\prime}$. For example, $1 \in \operatorname{supp} t_{n \varepsilon_{1}}\left(e^{\rho} R\right)$ for every $n \leq 0$ and so $\sum_{t \in T^{\prime \prime}} t\left(e^{\rho} R\right)$ is not well defined.

A similar argument applies for the cases $A(2 k, 2 k)^{(4)}$ and $D(k+1, k)^{(2)}$. However, we shall prove a stronger statement:

Lemma 5.3. For the cases $\hat{\mathfrak{g}}=A(2 k, 2 k)^{(4)}$ and $D(k+1, k)^{(2)}$, we have

$$
\mathcal{F}_{T^{\prime}}\left(e^{\rho} R\right)=\mathcal{F}_{\hat{W}^{\prime}}\left(\frac{e^{\rho}}{\prod_{\beta \in S}\left(1+e^{-\beta}\right)}\right)
$$

where $S$ is a maximal isotropic subset of $\pi$ and both sums are well defined elements of $\mathcal{R}$.

Proof. Let us show that the right hand side of (5.2) is well defined. For every $y \in \hat{W}^{\prime}$, we compute the maximal element $u(y)$ of the support of $y\left(e^{\rho} \prod_{\beta \in S}\left(1+e^{-\beta}\right)^{-1}\right)$ and see that each maximal element appears finitely many times and is less than or equal to $\max _{w \in W^{\prime}} w \rho$.

Write $y=t_{\mu} w$ where $t_{\mu} \in T^{\prime}$ and $w \in W^{\prime}$. Then

$$
\begin{aligned}
& u\left(t_{\mu} w\right) \quad=\quad t_{\mu} w \rho+\sum_{\beta \in S: t_{\mu} w \beta<0} t_{\mu} w \beta \\
& \rho=-\frac{1}{2} \sum_{\beta}{ }_{\beta \in S} \beta-\frac{1}{2} \sum_{\beta \in S}(w \beta-(w \beta, \mu) \delta)+\sum_{\beta \in S:(\mu, w \beta)>0}(w \beta-(w \beta, \mu) \delta)+\sum_{\beta \in S:(\mu, w \beta)=0, w \beta<0} w \beta \\
& =\quad w \rho+\sum_{\beta \in S: t_{\mu} w \beta<0} w \beta-\frac{1}{2} \sum_{\beta \in S}|(w \beta, \mu)| \delta .
\end{aligned}
$$

Hence $u\left(t_{\mu} w\right)=v^{\prime}-m \delta$ for $v^{\prime} \in Q$ only when $m=\frac{1}{2} \sum_{\beta \in S}|(w \beta, \mu)|$, which is possible only for finitely many $\mu$ 's.

Let us prove equality (5.2). In these cases, $\mathfrak{g}$ is isomorphic to $B(k, k)$ and the denominator identity holds for $W^{\prime}$ as well (see [G2, 2.2]), that is

$$
e^{\rho} R=\mathcal{F}_{W^{\prime}}\left(\frac{e^{\rho}}{\prod_{\beta \in S}\left(1+e^{-\beta}\right)}\right) .
$$

Since $\hat{W}^{\prime}=T^{\prime} \rtimes W^{\prime}$, the equality (5.2) follows.

5.3. Step II. In this step we show that $\hat{R}^{-1} e^{-\rho} \cdot \mathcal{F}_{T^{\prime}}\left(R e^{\rho}\right)$ takes the form $f(q)$. As in the nontwisted case [GR, 2.3.2], this follows from a proposition stating that $\operatorname{supp}(Y) \subset \hat{Q}^{\hat{W}}$. For all twisted affine Lie superalgebras $\hat{Q}^{\hat{W}}=\mathbb{Z} \delta$, completing this step of the proof. The proof of this proposition requires the following two lemmas.

Lemma 5.4. The term $e^{\hat{\rho}^{\prime}-\rho} \hat{R}_{\overline{1}} \cdot \mathcal{F}_{T^{\prime}}\left(e^{\rho} R\right)$ is a $\hat{W}^{\prime}$-anti-invariant element of $\mathcal{R}_{\hat{W}^{\prime}}$.

Proof. By Lemma 2.5, it suffices to find $Y \in \mathcal{R}_{\hat{W}}$, such that

$$
e^{\hat{\rho}^{\prime}-\rho} \hat{R}_{\overline{1}} \cdot \mathcal{F}_{T^{\prime}}\left(e^{\rho} R\right)=\mathcal{F}_{\hat{W}^{\prime}}(Y) .
$$

We will find $Y$ in the form $Y=e^{\hat{\rho}^{\prime}-\rho} \hat{R}_{\overline{1}} \cdot Z$. Since $e^{\hat{\rho}^{\prime}-\rho} \hat{R}_{\overline{1}}$ is $\hat{W}^{\prime}$-invariant (see Remark 2.7), the equality (5.3) is equivalent to

$$
\mathcal{F}_{T^{\prime}}\left(e^{\rho} R\right)=\mathcal{F}_{\hat{W}^{\prime}}(Z)
$$


For the cases $A(2 k, 2 k)^{(4)}$ and $D(k+1, k)^{(2)}$, we take $Z:=e^{\rho} \prod_{\beta \in S}\left(1+e^{-\beta}\right)^{-1}$. Then, equality (5.4) follows from Lemma 5.3 and $Y=e^{\hat{\rho}^{\prime}-\rho} \hat{R}_{\overline{1}} \cdot e^{\rho} \prod_{\beta \in S}\left(1+e^{-\beta}\right)^{-1}$ belongs to $\mathcal{R}_{\hat{W}}$, by Section 2.4

For the remaining $A(2 k-1,2 k-1)^{(2)}$ case, we take $Z:=e^{\rho} R_{\overline{0}}^{\prime \prime} \cdot R_{\overline{1}}^{-1}$ where $R_{\overline{0}}^{\prime \prime}=\prod_{\alpha \in \Delta^{\prime \prime+}}\left(1-e^{-\alpha}\right)$. Then $Y=e^{\hat{\rho}^{\prime}} \hat{R}_{\overline{1}} \cdot R_{\overline{0}}^{\prime \prime} \cdot R_{\overline{1}}^{-1}$ is again in $\mathcal{R}_{\hat{W}^{\prime}}$, by Section 2.4. Let us prove equality (5.4). Note that $e^{\rho} R_{\overline{0}}^{\prime \prime}$ is $\hat{W}^{\prime}$-invariant and $\rho=0$. Dividing both sides of equality (5.4) by $e^{\rho} R_{\overline{0}}^{\prime \prime}$ we obtain the equivalent equality

$$
\mathcal{F}_{T^{\prime}}\left(R_{\overline{0}}^{\prime} \cdot R_{\overline{1}}^{-1}\right)=\mathcal{F}_{\hat{W}^{\prime}}\left(R_{\overline{1}}^{-1}\right) .
$$

Note that the left hand side of (5.5) belongs to $\mathcal{R}^{\prime}$ because $\mathcal{F}_{T^{\prime}}\left(e^{\rho} R\right) \in \mathcal{R}$.

Let us show that $\mathcal{F}_{\hat{W}^{\prime}}\left(R_{\overline{1}}^{-1}\right)$ is well defined and belongs to $\mathcal{R}$. For every $y \in \hat{W}^{\prime}$, one has

$$
\operatorname{maxsupp}\left(y R_{\overline{1}}^{-1}\right)=\sum_{\beta \in \Delta_{\overline{1}}^{+}: y \beta<0} y \beta .
$$

Let us show that for every $\nu \in-\hat{Q}^{+}$, there are only finitely many $y \in \hat{W}^{\prime}$ such that maxsupp $\left(y R_{\overline{1}}^{-1}\right)=$ $\nu$. Write $y=t_{\mu} w$ where $t_{\mu} \in T^{\prime}$ and $w \in W^{\prime}$. Note that $y \beta<0$ if $(\mu, w \beta)>0$ and that $(\mu, w \beta)=0$ implies that $y \beta \in Q$. Since $\mu \in \operatorname{span}\left\{\delta_{i}\right\}_{i=1}^{k}$, for every $i=1, \ldots k$ one has either $\left(\mu, w\left(\varepsilon_{i}-\delta_{i}\right)\right)>0$ or $\left(\mu, w\left(\varepsilon_{i}+\delta_{i}\right)\right)>0$. Write $\nu=-m \delta+\nu^{\prime}$ where $\nu^{\prime} \in Q$ and $\mu=\sum_{i=1}^{k} a_{i} \delta_{i}$. We get that $\sum_{i=1}^{k}\left|a_{i}\right| \leq m$ which is possible only for finitely many $\mu$ 's. Thus, the sum $\mathcal{F}_{\hat{W}^{\prime}}\left(R_{\overline{1}}^{-1}\right)$ is well defined.

We are left to verify equality (5.5). Recall that in this case $\hat{W}^{\prime} \nsupseteq T^{\prime}$. The groups $\hat{W}^{\prime}$ and $W^{\prime}$ are extended to $\hat{W}_{C_{k}}=\left\langle\hat{W}^{\prime}, s_{\delta_{k}}\right\rangle$ and $W_{C_{k}}=\left\langle W^{\prime}, s_{\delta_{k}}\right\rangle$, respectively, and $\hat{W}_{C_{k}}=T^{\prime} \rtimes W_{C_{k}}$. Recall that the sign function is extended from $\hat{W}^{\prime}$ to $\hat{W}_{C_{k}}$ such that $\operatorname{sgn} s_{\delta_{k}}=1$ (see 2.3). One has

$$
\mathcal{F}_{\hat{W}_{C_{k}}}\left(R_{\overline{1}}^{-1}\right)=\mathcal{F}_{\hat{W}_{C_{k}}}\left(R_{\overline{1}}^{-1}\right)+\mathcal{F}_{\hat{W}^{\prime}} s_{\delta_{k}}\left(R_{\overline{1}}^{-1}\right)=2 \mathcal{F}_{\hat{W}^{\prime}}\left(R_{\overline{1}}^{-1}\right) .
$$

Since $\rho=0, e^{\rho^{\prime}} R_{1}$ is $W_{C_{k}}$-invariant (see Remark 2.7). We obtain

$$
\begin{aligned}
\mathcal{F}_{\hat{W}_{C_{k}}}\left(R_{\overline{1}}^{-1}\right) & =\mathcal{F}_{T^{\prime}}\left(\mathcal{F}_{W_{C_{k}}}\left(R_{\overline{1}}^{-1}\right)\right) \\
& =\mathcal{F}_{T^{\prime}}\left(e^{-\rho^{\prime}} R_{\overline{1}}^{-1} \cdot \mathcal{F}_{W_{C_{k}}}\left(e^{\rho^{\prime}}\right)\right) \\
& =\mathcal{F}_{T^{\prime}}\left(e^{-\rho^{\prime}} R_{\overline{1}}^{-1} \cdot \mathcal{F}_{W^{\prime}}\left(e^{\rho^{\prime}}+s_{\delta_{k}} e^{\rho^{\prime}}\right)\right) \\
& =2 \mathcal{F}_{T^{\prime}}\left(R_{\overline{0}}^{\prime} \cdot R_{\overline{1}}^{-1}\right)
\end{aligned}
$$

as required. $\mathrm{B}$

Lemma 5.5. For $\hat{\mathfrak{g}}=A(2 k, 2 k)^{(4)}$ or $D(k+1, k)^{(2)}$, the term $e^{\hat{\rho}^{\prime \prime}-\rho} \hat{R}_{\overline{1}} \cdot \mathcal{F}_{T^{\prime}}\left(e^{\rho} R\right)$ is a $\hat{W}^{\prime \prime}$-antiinvariant element of $\mathcal{R}_{\hat{W}^{\prime \prime}}$.

Proof. Note that $e^{\hat{\rho}^{\prime}-\rho} \hat{R}_{\overline{1}}$ is $\hat{W}^{\prime}$-invariant and by Section 2.4

$$
e^{\hat{\rho}^{\prime \prime}-\rho} \hat{R}_{\overline{1}} \cdot \mathcal{F}_{T^{\prime}}\left(e^{\rho} R\right)=e^{\hat{\rho}^{\prime \prime}-\hat{\rho}^{\prime}} \mathcal{F}_{T^{\prime}}\left(e^{\hat{\rho}^{\prime}} \hat{R}_{\overline{1}} \cdot R\right) .
$$

Let us show $\hat{W}^{\prime \prime}$-anti-invariance. The term $e^{\hat{\rho}^{\prime \prime}-\rho} \hat{R}_{\overline{1}}$ is $\hat{W}^{\prime \prime}$-invariant (Remark 2.77) and so it suffices to show that $\mathcal{F}_{T^{\prime}}\left(e^{\rho} R\right)$ is $\hat{W}^{\prime \prime}$-anti-invariant. Note that $\hat{W}^{\prime}$ and $\hat{W}^{\prime \prime}$ commute. The anti-invariance with respect to $W^{\prime \prime}$ follows from the one of $e^{\rho} R$. It remains to show invariance with respect to 
$T^{\prime \prime}$. By Lemma 5.3, $\mathcal{F}_{T^{\prime}}\left(e^{\rho} R\right)=\mathcal{F}_{\hat{W}^{\prime}}\left(e^{\rho} \prod_{\beta \in S}\left(1+e^{-\beta}\right)^{-1}\right)$. Since $\rho=\frac{1}{2} \sum_{i=1}^{k}\left(\delta_{i}-\varepsilon_{i}\right)$ and $S=\left\{\varepsilon_{i}-\delta_{i}\right\}_{i=1}^{k}$,

$$
t_{\sum 2 n_{i} \varepsilon_{i}}\left(e^{\rho} \prod_{\beta \in S}\left(1+e^{-\beta}\right)^{-1}\right)=t_{\sum 2 n_{i} \delta_{i}}\left(e^{\rho} \prod_{\beta \in S}\left(1+e^{-\beta}\right)^{-1}\right)
$$

and the assertion follows.

Proposition 5.6. The support of $e^{-\rho} \hat{R}^{-1} \cdot \mathcal{F}_{T^{\prime}}\left(e^{\rho} R\right)$ is contained in $\mathbb{Z} \delta$.

Proof. We generalize the argument of [GR, 2.3.2]. Let $Y:=e^{-\rho} \hat{R}^{-1} \cdot \mathcal{F}_{T^{\prime}}\left(e^{\rho} R\right)$. Note that $e^{\hat{\rho}^{\prime}-\rho} \hat{R}_{\overline{1}}$. $\mathcal{F}_{T^{\prime}}\left(e^{\rho} R\right)=e^{\hat{\rho}^{\prime}} \hat{R}_{\overline{0}} \cdot Y$ which is a $\hat{W}^{\prime}$-anti-invariant element of $\mathcal{R}_{\hat{W}^{\prime}}$ by Lemma 5.4. Write $Y=Y_{1}+Y_{2}$ where $\operatorname{supp}\left(Y_{1}\right) \subset \mathbb{Z} \delta$ and $\operatorname{supp}\left(Y_{2}\right) \cap \mathbb{Z} \delta=\emptyset$. Since $e^{\hat{\rho}^{\prime}} \hat{R}_{\overline{0}}$ and $Y_{1}$ are $\hat{W}$-anti-invariant and invariant, respectively, the term

$$
e^{\hat{\rho}^{\prime}} \hat{R}_{\overline{0}} \cdot Y_{2}=e^{\hat{\rho}^{\prime}} \hat{R}_{\overline{0}} \cdot Y-e^{\hat{\rho}^{\prime}} \hat{R}_{\overline{0}} \cdot Y_{1}
$$

is also a $\hat{W}^{\prime}$-anti-invariant element of $\mathcal{R}_{\hat{W}^{\prime}}$. We would like to show that this term is equal to zero. Let us show that every maximal element $\mu \in \operatorname{supp}\left(Y_{2}\right)$ belongs to $\mathbb{Z} \delta$, in contradiction to the definition of $Y_{2}$. The element $\mu+\hat{\rho}^{\prime}$ is maximal in $\operatorname{supp}\left(\hat{R}_{\overline{0}} e^{\hat{\rho}^{\prime}} \cdot Y_{2}\right)$. By Lemma 2.6, $\operatorname{supp}\left(\hat{R}_{\overline{0}} e^{\hat{\rho}^{\prime}} \cdot Y_{2}\right)$ is a union of regular orbits. Since $\mu+\hat{\rho}^{\prime}$ is maximal in a regular $\hat{W}^{\prime}$-orbit and $\frac{2\left(\hat{\rho}^{\prime}, \alpha\right)}{(\alpha, \alpha)}=1$ for all $\alpha \in \hat{\pi}^{\prime}$, one has $(\mu, \alpha) \geq 0$. On the other hand, $(\mu, \delta)=0$ and $\delta \in \mathbb{Z}_{>0} \hat{\pi}^{\prime}$ and hence $\left(\mu, \hat{\pi}^{\prime}\right)=0$. Since $\mathbb{Z} \hat{\Delta}^{\prime \prime}$ is the orthogonal set to $\hat{\pi}^{\prime}$ in $\hat{Q}$, we get that $\mu \in \mathbb{Z} \hat{\Delta}^{\prime \prime}$.

For the cases $A(2 k, 2 k)^{(4)}$ and $D(k+1, k)^{(2)}$, we can interchange $\hat{W}^{\prime}$ by $\hat{W}^{\prime \prime}$ and apply the same argument (using Lemma [5.5 instead of Lemma [5.4). Thus, $\mu \in \hat{\Delta}^{\prime} \cap \hat{\Delta}^{\prime \prime}$ and hence $\mu \in \mathbb{Z} \delta$.

For the case $A(2 k-1,2 k-1)^{(2)}$, let us show that the support of $\mu+\rho$ belongs to $U$. Note that $\hat{R} e^{\rho} Y_{2}=\mathcal{F}_{T^{\prime}}\left(R e^{\rho}\right)-\hat{R} e^{\rho} Y_{1}$ and by Section 3 ,

$$
\operatorname{supp}\left(\mathcal{F}_{T^{\prime}}\left(R e^{\rho}\right)\right), \operatorname{supp}\left(\hat{R} e^{\rho}\right) \subset U .
$$

Since $(\delta, \rho)=(\delta, \hat{Q})=(\delta, \delta)=0, U+\mathbb{Z} \delta \subset U$. Hence, $\operatorname{supp}\left(\hat{R} e^{\rho} Y_{1}\right) \subset U$ implying that $\operatorname{supp}\left(\hat{R} e^{\rho} Y_{2}\right) \subset U$ and so $\mu+\rho \in U$.

Thus, $(\mu+\rho, \mu+\rho)=(\rho, \rho)=0$. This is equivalent to $(\mu, \mu)=0$ because $\rho=0$. Since the bilinear form is negative definite on $\Delta^{\prime \prime}$, we get that $\mu \in \mathbb{Z} \delta$ and the proposition follows.

Remark 5.7. When $h^{\vee} \neq 0$ we can not use this argument since $(\delta, \hat{\rho}) \neq 0$ and $\operatorname{supp}\left(\hat{R} e^{\hat{\rho}} Y_{1}\right) \not \subset U$.

5.4. Step III. In this section we compute $\mathcal{F}_{T^{\prime}}\left(e^{\rho} R\right) \cdot e^{-\rho} \hat{R}^{-1}$, knowing that it depends only on $q$, we describe an evaluation of the variables $e^{-\alpha}, \alpha \in \pi$, in which $\mathcal{F}_{T^{\prime}}\left(e^{\rho} R\right) \cdot e^{-\rho} R^{-1}$ is equal to 1 and $R \cdot \hat{R}^{-1}$ can be easily computed. We use the property of the algebras $A(2 k-1,2 k-1)^{(2)}$, $A(2 k, 2 k)^{(4)}$ and $D(k+1, k)^{(2)}$ that $\left|\Delta_{\overline{1}}^{+}\right|-\left|\Delta_{\overline{0}}^{+}\right|$is equal to the defect which is $k$.

Let $x \in \mathbb{C} \backslash\{0\}$ and evaluate $e^{-\alpha}$ by $(-1)^{p(\alpha)} \cdot x$ for every $\alpha \in \pi$, where $p(\alpha) \in\{\overline{0}, \overline{1}\}$ denotes the parity of $\alpha$. It implies that $e^{-\gamma}$ is evaluated by $(-1)^{p(\gamma)} x^{\text {ht }(\gamma)}$ for every $\gamma \in \Delta$.

Lemma 5.8. Let $\hat{\mathfrak{g}}$ be one of the algebras $A(2 k-1,2 k-1)^{(2)}, A(2 k, 2 k)^{(4)}$ and $D(k+1, k)^{(2)}$. Then for every $t \in T^{\prime}, t \neq i d,\left.\frac{\left(t\left(e^{\rho} R\right)\right)(x)}{\left(e^{\rho} R\right)(x)}\right|_{x=1}$ is equal to 0 . 
Proof. One has that $e^{\rho}(x)=x^{n}$ for some $n \in \frac{1}{2} \mathbb{Z}$ and

$$
R(x)=\frac{\prod_{\gamma \in \Delta_{\overline{0}}^{+}}\left(1-x^{\mathrm{ht} \gamma}\right)}{\prod_{\gamma \in \Delta_{\overline{1}}^{+}}\left(1-x^{\mathrm{ht} \gamma}\right)}
$$

and hence at $x=1$, the function $e^{\rho} R(x)$ has a pole of order $\left|\Delta_{\overline{1}}^{+}\right|-\left|\Delta_{\overline{0}}^{+}\right|=k$.

Let us show that if $t_{\mu} \neq i d$, then $\left(t_{\mu}\left(e^{\rho} R\right)\right)(x)$ has a pole at $x=1$ of order which is strictly less than $k$. By the denominator identity of finite dimensional Lie superalgebras (see (2.3))

$$
t_{\mu}\left(e^{\rho} R\right)=\sum_{w \in W^{\prime \prime}}(-1)^{l(w)} \frac{e^{t_{\mu} w \rho}}{\prod_{i=1}^{k}\left(1+e^{-t_{\mu} w \beta_{i}}\right)}
$$

One has $t_{\mu} w \beta_{i}=w \beta_{i}+n_{i} \delta$ where $n_{i}=\left(\mu, w \beta_{i}\right) \in \mathbb{Z}$. Hence the evaluation of $\left(1+e^{-t w \beta_{i}}\right)^{-1}$ is equal to $\left(1-x^{m} q^{n_{i}}\right)^{-1}$ for some $m \in \mathbb{Z}_{\neq 0}$. Hence it has a pole at $x=1$ if and only if $n_{i}=0$. Thus, the evaluation of $t_{\mu}\left(e^{\rho} R\right)$ has a pole of order less or equal to the number of $n_{i}$ 's which are zero. This number is equal to $k$ if and only if $\left(\mu, \delta_{i}\right)=0$ for $i=1, \ldots, k$. Since $\mu \in \operatorname{span}\left\{\delta_{1}, \ldots, \delta_{k}\right\}$, we get that $\mu=0$, that is $t=i d$ and the assertion follows.

Remark 5.9. The above argument is based on the fact that the rank of $T^{\prime}$ is equal to the defect. In particular, it can not be used for the case $h^{\vee} \neq 0$.

Let us compute $\left.\frac{\hat{R}}{R}(x)\right|_{x=1}$.

Case $A(2 k-1,2 k-1)^{(2)}$ : One has

$$
\frac{\hat{R}}{R}=\prod_{n=1}^{\infty} \frac{\prod_{\alpha \in \Delta_{\overline{0}}^{(n \bmod 2)}}\left(1-q^{n} e^{\alpha}\right)\left(1-q^{2 n}\right)^{\operatorname{dim} \mathfrak{h}}\left(1-q^{2 n+1}\right)^{\operatorname{dim} \hat{\mathfrak{g}}_{\delta}}}{\prod_{\alpha \in \Delta_{\overline{1}}^{(n \bmod 2)}}\left(1+q^{n} e^{\alpha}\right)} .
$$

Here $\left|\Delta_{\overline{0}}^{(0)}\right|=\left|\Delta_{\overline{0}}^{(1)}\right|=4 k^{2}-2 k,\left|\Delta_{\overline{1}}^{(0)}\right|=\left|\Delta_{\overline{1}}^{(1)}\right|=4 k^{2}, \operatorname{dim} \mathfrak{h}=2 k$ and $\operatorname{dim} \hat{\mathfrak{g}}_{\delta}=2 k-2$. So

$$
\left.\frac{\hat{R}}{R}(x)\right|_{x=1}=\prod_{n=1}^{\infty}\left(1-q^{2 n+1}\right)^{-2} .
$$

Case $A(2 k, 2 k)^{(4)}$ : One has

$$
\frac{\hat{R}}{R}=\prod_{n=1}^{\infty} \frac{\prod_{\alpha \in \Delta_{\overline{0}}^{(n \bmod 4)}}\left(1-q^{n} e^{\alpha}\right)\left(1-q^{4 n}\right)^{\operatorname{dim} \mathfrak{h}}\left(1-q^{4 n+2}\right)^{\operatorname{dim} \hat{\mathfrak{g}}_{2 \delta}}}{\prod_{\alpha \in \Delta_{\overline{1}}^{(n \bmod 4)}}\left(1+q^{n} e^{\alpha}\right)\left(1+q^{4 n+1}\right)^{\operatorname{dim} \hat{\mathfrak{g}}_{\delta}}\left(1+q^{4 n+3}\right)^{\operatorname{dim} \hat{\mathfrak{g}}_{3 \delta}}} .
$$

Here $\left|\Delta_{\overline{0}}^{(0)}\right|=\left|\Delta_{\overline{0}}^{(2)}\right|=4 k^{2},\left|\Delta_{\overline{0}}^{(1)}\right|=\left|\Delta_{\overline{0}}^{(3)}\right|=2 k,\left|\Delta_{\overline{1}}^{(0)}\right|=\left|\Delta_{\overline{1}}^{(2)}\right|=4 k^{2}+2 k,\left|\Delta_{\overline{1}}^{(1)}\right|=\left|\Delta_{\overline{1}}^{(3)}\right|=2 k$, $\operatorname{dim} \mathfrak{h}=\operatorname{dim} \hat{\mathfrak{g}}_{2 \delta}=2 k$ and $\operatorname{dim} \hat{\mathfrak{g}}_{\delta}=\operatorname{dim} \hat{\mathfrak{g}}_{3 \delta}=1$. So

$$
\left.\frac{\hat{R}}{R}(x)\right|_{x=1}=\prod_{n=1}^{\infty}\left(1+q^{2 n+1}\right)^{-1} .
$$

Case $D(k+1, k)^{(2)}$ : One has

$$
\frac{\hat{R}}{R}=\prod_{n=1}^{\infty} \frac{\prod_{\alpha \in \Delta_{\overline{0}}^{(n \bmod 2)}}\left(1-q^{n} e^{\alpha}\right)\left(1-q^{2 n}\right)^{\operatorname{dim} \mathfrak{h}}\left(1-q^{2 n+1}\right)^{\operatorname{dim} \hat{\mathfrak{g}}_{\delta}}}{\prod_{\alpha \in \Delta_{\overline{1}}^{(n \bmod 2)}}\left(1+q^{n} e^{\alpha}\right)} .
$$


Here $\left|\Delta_{\overline{0}}^{(0)}\right|=4 k^{2},\left|\Delta_{\overline{0}}^{(1)}\right|=\left|\Delta_{\overline{1}}^{(1)}\right|=2 k,\left|\Delta_{\overline{1}}^{(0)}\right|=4 k^{2}+2 k, \operatorname{dim} \mathfrak{h}=2 k$ and $\operatorname{dim} \hat{\mathfrak{g}}_{\delta}=1$. So

$$
\left.\frac{\hat{R}}{R}(x)\right|_{x=1}=\prod_{n=1}^{\infty}\left(1-q^{2 n+1}\right) .
$$

Remark 5.10. For the computation of $\operatorname{dim} \mathfrak{g}_{\delta}$ see [vdL2, 7.5.13] or note that for $\tilde{\mathfrak{g}}=A(2 k-1,2 k-1)$, $D(k+1, k)$,

$$
\begin{aligned}
\operatorname{dim} \tilde{\mathfrak{g}}_{\overline{0}} & =\left|\Delta_{\overline{0}}^{(0)}\right|+\left|\Delta_{\overline{0}}^{(1)}\right|+\operatorname{dim} \mathfrak{h}+\operatorname{dim} \hat{\mathfrak{g}}_{\delta} \\
\operatorname{dim} \tilde{\mathfrak{g}}_{\overline{1}} & =\left|\Delta_{\overline{1}}^{(0)}\right|+\left|\Delta_{\overline{1}}^{(1)}\right|
\end{aligned}
$$

and for $\tilde{\mathfrak{g}}=A(2 k, 2 k)$,

$$
\begin{aligned}
\operatorname{dim} \tilde{\mathfrak{g}}_{\overline{0}} & =\left|\Delta_{\overline{0}}^{(0)}\right|+\left|\Delta_{\overline{0}}^{(1)}\right|+\left|\Delta_{\overline{0}}^{(2)}\right|+\left|\Delta_{\overline{0}}^{(3)}\right|+\operatorname{dim} \mathfrak{h}+\operatorname{dim} \hat{\mathfrak{g}}_{2 \delta} \\
\operatorname{dim} \tilde{\mathfrak{g}}_{\overline{1}} & =\left|\Delta_{\overline{1}}^{(0)}\right|+\left|\Delta_{\overline{1}}^{(1)}\right|+\left|\Delta_{\overline{1}}^{(2)}\right|+\left|\Delta_{\overline{1}}^{(3)}\right|+\operatorname{dim} \hat{\mathfrak{g}}_{\delta}+\operatorname{dim} \hat{\mathfrak{g}}_{3 \delta} .
\end{aligned}
$$

\section{Appendix}

We recall the construction of the twisted affine Lie superalgebras and the description of their root systems. We list choices of simple roots which we use to prove the denominator identity.

6.1. A construction of the twisted affine Lie superalgebras. We describe the automorphisms which are used in vdL2 to construct the twisted affine Lie superalgebras. For every algebra we show how the automorphism acts on the Chevalley generators $e_{\alpha_{i}}, f_{\alpha_{i}}$ with respect to a standard choice of simple roots $\pi=\left\{\alpha_{1}, \ldots, \alpha_{n}\right\}$ (a choice that contains at most one isotropic root). For $\alpha \in \tilde{\Delta}^{+}$, let $e_{\alpha}:=\left[e_{\alpha_{i_{1}}},\left[e_{\alpha_{i_{2}}}, \ldots\left[e_{\alpha_{i_{m-1}}}, e_{\alpha_{i_{m}}}\right]\right]\right]$ where $\alpha_{i} \in \tilde{\pi}$ and $\alpha=\alpha_{i_{1}}+\cdots+\alpha_{i_{m}}, i_{1} \leq \ldots \leq i_{m}$. For $\alpha \in \tilde{\Delta}^{-}$define $f_{\alpha}$ similarly.

If for all $i=1, \ldots, n, \sigma\left(e_{\alpha_{i}}\right)$ is a scalar multiple of $e_{\alpha_{j}}$ for some $\alpha_{j} \in \tilde{\pi}$, we call $\sigma$ an almostdiagram automorphism and denote $\sigma\left(\alpha_{i}\right):=\alpha_{j}$. For Lie algebras, all finite order automorphisms are conjugated to almost diagram automorphisms and all twisted affine Lie algebras can be defined using a diagram automorphism (with no scalar multiples). We show that $A(2 k, 2 l)^{(4)}$ can not be defined using an almost-diagram automorphism.

6.1.1. $A(2 k, 2 l-1)^{(2)}$. Let us define an automorphism $\sigma$ of order 2 on $A(2 k, 2 l-1)$. Take

$$
\tilde{\pi}=\left\{\varepsilon_{1}-\varepsilon_{2}, \ldots, \varepsilon_{2 k}-\varepsilon_{2 k+1}, \varepsilon_{2 k+1}-\delta_{1}, \delta_{1}-\delta_{2}, \ldots, \delta_{2 l-1}-\delta_{2 l}\right\} .
$$

Then $\sigma$ is defined by

$$
\begin{array}{lll}
\sigma\left(e_{\varepsilon_{i}-\varepsilon_{i+1}}\right)=e_{\varepsilon_{2 k+1-i}-\varepsilon_{2 k+2-i}}, & \sigma\left(e_{\delta_{i}-\delta_{i+1}}\right)=e_{\delta_{2 l-i}-\delta_{2 l+1-i}}, & \sigma\left(e_{\varepsilon_{2 k+1}-\delta_{1}}\right)=f_{\varepsilon_{1}-\delta_{2 l}}, \\
\sigma\left(f_{\varepsilon_{i}-\varepsilon_{i+1}}\right)=f_{\varepsilon_{2 k+1-i}-\varepsilon_{2 k+2-i}}, & \sigma\left(f_{\delta_{i}-\delta_{i+1}}\right)=f_{\delta_{2 l-i}-\delta_{2 l+1-i}}, & \sigma\left(f_{\varepsilon_{2 k+1}-\delta_{1}}\right)=-e_{\varepsilon_{1}-\delta_{2 l}} .
\end{array}
$$


6.1.2. $A(2 k-1,2 l-1)^{(2)}$. Let us define an automorphism $\sigma$ of order 2 on $A(2 k-1,2 l-1)$ (i.e. $\mathfrak{p s l}(2 k, 2 k)$ if $k=l)$. Take

$$
\tilde{\pi}=\left\{\varepsilon_{1}-\varepsilon_{2}, \ldots, \varepsilon_{2 k-1}-\varepsilon_{2 k}, \varepsilon_{2 k}-\delta_{1}, \delta_{1}-\delta_{2}, \ldots, \delta_{2 l-1}-\delta_{2 l}\right\} .
$$

Then $\sigma$ is defined by

$$
\begin{array}{lll}
\sigma\left(e_{\varepsilon_{i}-\varepsilon_{i+1}}\right)=e_{\varepsilon_{2 k-i}-\varepsilon_{2 k+1-i}} \cdot(-1)^{-\delta_{i, k}}, & \sigma\left(e_{\delta_{i}-\delta_{i+1}}\right)=e_{\delta_{2 l-i}-\delta_{2 l+1-i}}, & \sigma\left(e_{\varepsilon_{2 k+1}-\delta_{1}}\right)=f_{\varepsilon_{1}-\delta_{2 l}}, \\
\sigma\left(f_{\varepsilon_{i}-\varepsilon_{i+1}}\right)=f_{\varepsilon_{2 k-i}-\varepsilon_{2 k+1-i}} \cdot(-1)^{-\delta_{i, k}}, & \sigma\left(f_{\delta_{i}-\delta_{i+1}}\right)=f_{\delta_{2 l-i}-\delta_{2 l+1-i}}, & \sigma\left(f_{\varepsilon_{2 k+1}-\delta_{1}}\right)=-e_{\varepsilon_{1}-\delta_{2 l}} .
\end{array}
$$

6.1.3. $A(2 k, 2 l)^{(4)}$. Let us define an automorphism $\sigma$ of order 4 on $A(2 k, 2 l)($ i.e. $\mathfrak{p s l}(2 k+1,2 k+1)$ if $k=l$ ). Take

$$
\tilde{\pi}=\left\{\varepsilon_{1}-\varepsilon_{2}, \ldots, \varepsilon_{2 k}-\varepsilon_{2 k+1}, \varepsilon_{2 k+1}-\delta_{1}, \delta_{1}-\delta_{2}, \ldots, \delta_{2 l}-\delta_{2 l+1}\right\} .
$$

Then $\sigma$ is defined as for $A(2 k, 2 l-1)$ on $\left\{\varepsilon_{1}-\varepsilon_{2}, \ldots, \varepsilon_{2 k}-\varepsilon_{2 k+1}, \varepsilon_{2 k+1}-\delta_{1}, \delta_{1}-\delta_{2}, \ldots, \delta_{2 l-1}-\delta_{2 l}\right\}$ and

$$
\begin{aligned}
& \sigma\left(e_{\delta_{2 l}-\delta_{2 l+1}}\right)=-i \cdot f_{\delta_{1}-\delta_{2 l+1}} \\
& \sigma\left(f_{\delta_{2 l}-\delta_{2 l+1}}\right)=i \cdot e_{\delta_{1}-\delta_{2 l+1}} .
\end{aligned}
$$

Proposition 6.1. The algebra $A(2 k, 2 l)^{(4)}$ can not be defined using an almost-diagram automorphism.

Proof. Let $\mathfrak{h}:=\mathfrak{g} \cap \tilde{\mathfrak{h}}$, where $\mathfrak{g}$ is the algebra formed by the fixed points of $\sigma$ and $\tilde{\mathfrak{h}}$ the Cartan subalgebra of $\tilde{\mathfrak{g}}=A(2 k, 2 l)$. Note that $e_{\varepsilon_{k+1}-\delta_{2 l+1}}$ and $f_{\varepsilon_{k+1}-\delta_{2 l+1}}$ commute with $\mathfrak{h}$. This gives rise to the imaginary odd root $\delta$ of $\hat{\mathfrak{g}}$ with the root vector $t \otimes\left(e_{\varepsilon_{k+1}-\delta_{2 l+1}}+f_{\varepsilon_{k+1}-\delta_{2 l+1}}\right)$. Let us show that this situation can not occur for almost-diagram automorphisms.

Suppose $\sigma$ is an almost diagram automorphism. Then it would permute the fundamental co-roots $\varpi_{\alpha}$, since if $\left[\varpi_{\alpha}, e_{\beta}\right]=\delta_{\alpha, \beta} e_{\beta}$ then $\left[\sigma\left(\varpi_{\alpha}\right), e_{\sigma(\beta)}\right]=\delta_{\sigma(\alpha), \sigma(\beta)} e_{\sigma(\beta)}$. Hence $h:=\sum \varpi_{\alpha}$ belongs to $\mathfrak{h}$. However $h$ is a regular element of $\tilde{\mathfrak{g}}$, that is $\left[h, e_{\gamma}\right]=\mathrm{ht}(\gamma) e_{\gamma},\left[h, f_{\gamma}\right]=-\mathrm{ht}(\gamma) f_{\gamma}$. Thus, the centralizer of $\mathfrak{h}$ in $\tilde{\mathfrak{g}}$ is $\tilde{\mathfrak{h}}$, in particular, there are no imaginary odd roots.

6.1.4. $D(k+1, l)^{(2)}$ and $C(k+1)^{(2)}$. Let us define an automorphism $\sigma$ of order 2 on $D(k+1, l)$ and $C(k+1)$. For $D(k+1, l)$ take

$$
\tilde{\pi}=\left\{\varepsilon_{1}-\varepsilon_{2}, \ldots, \varepsilon_{k}-\varepsilon_{k+1}, \varepsilon_{k+1}-\delta_{1}, \delta_{1}-\delta_{2}, \ldots, \delta_{l-1}-\delta_{l}, 2 \delta_{l}\right\}
$$

and for $C(k+1)$ take

$$
\tilde{\pi}=\left\{\varepsilon_{1}-\varepsilon_{2}, \ldots, \varepsilon_{k}-\varepsilon_{k+1}, \varepsilon_{k+1}-\delta_{1}\right\} .
$$

The automorphism $\sigma$ acts by

$$
\begin{array}{ll}
\sigma\left(e_{\varepsilon_{k}-\varepsilon_{k+1}}\right)=e_{\varepsilon_{k}+\varepsilon_{k+1}}, & \sigma\left(e_{\varepsilon_{k+1}-\delta_{1}}\right)=f_{\varepsilon_{k+1}+\delta_{1}}, \\
\sigma\left(f_{\varepsilon_{k}-\varepsilon_{k+1}}\right)=f_{\varepsilon_{k}+\varepsilon_{k+1}}, & \sigma\left(f_{\varepsilon_{k+1}-\delta_{1}}\right)=e_{\varepsilon_{k+1}-\delta_{1}} .
\end{array}
$$

fixing the rest of the Chevalley generators.

Remark 6.2. The automorphism $\sigma$ is a diagram automorphism with respect to

$$
\tilde{\pi}=\left\{\varepsilon_{1}-\varepsilon_{2}, \ldots, \varepsilon_{k-1}-\varepsilon_{k}, \varepsilon_{k}-\delta_{1}, \delta_{1}-\delta_{2}, \ldots, \delta_{l-1}-\delta_{l}, \delta_{l}-\varepsilon_{k+1}, \delta_{l}+\varepsilon_{k+1}\right\} .
$$


6.1.5. $G(3)^{(2)}$. Let us define an automorphism $\sigma$ of order 2 on $G(3)$. Take

$$
\tilde{\pi}=\left\{\varepsilon_{3}-\varepsilon_{2}, \varepsilon_{2}-\delta_{1}, \delta_{1}\right\}
$$

Then $\sigma$ is defined by

$$
\begin{aligned}
& \sigma\left(e_{\delta_{1}}\right)=-e_{\delta_{1}}, \\
& \sigma\left(f_{\delta_{1}}\right)=-f_{\delta_{1}} .
\end{aligned}
$$

and fixing the rest of the Chevalley generators.

6.2. Description of the root systems of the twisted affine Lie superalgebras and choices of simple roots. In this section, we describe the root systems of the twisted affine Lie superalgebras for which we prove the denominator identity, see Table 1 . The root systems are described in terms of a basis $\left\{\varepsilon_{i}, \delta_{j}, \delta \mid 1 \leq i \leq k, 1 \leq j \leq l\right\}$. The bilinear form $(\cdot, \cdot)$ defined by

$$
\left(\varepsilon_{i}, \varepsilon_{j}\right)=-\left(\delta_{i}, \delta_{j}\right)=\delta_{i j}, \quad\left(\varepsilon_{i}, \delta_{j}\right)=0
$$

when $h^{\vee} \neq 0$ and $\hat{\mathfrak{g}} \neq G(3)^{(2)}$. When $h^{\vee}=0$ we have

$$
\left(\varepsilon_{i}, \varepsilon_{j}\right)=-\left(\delta_{i}, \delta_{j}\right)=-\delta_{i j}, \quad\left(\varepsilon_{i}, \delta_{j}\right)=0
$$

The root system of $G(3)^{(2)}$ is described by the basis $\left\{\varepsilon_{1}, \varepsilon_{2}, \varepsilon_{3}\right\}$ and the inner product is defined such that $\left(\varepsilon_{1}, \varepsilon_{1}\right)=1 \frac{1}{2},\left(\varepsilon_{2}, \varepsilon_{2}\right)=\frac{1}{2},\left(\varepsilon_{3}, \varepsilon_{3}\right)=-2$ and $\left(\varepsilon_{i}, \varepsilon_{j}\right)=0$ if $i \neq j$. Here $\delta$ denotes the minimal imaginary root.

In Table 2 we present a choice of simple roots which is used to prove Theorem 1.1 for each root system of a twisted affine Lie superalgebra. In Table 3 we list the types of the finite part and $\hat{\Delta}^{\prime}$ and $\hat{\Delta}^{\prime \prime}$ of each algebra. 
Table 1. Root systems

\begin{tabular}{lll}
\hline The algebra & & Roots \\
\hline$A(2 k, 2 l-1)^{(2)}$ & $k \geq l$ & $\hat{\Delta}_{\overline{0}}=\left\{s \delta_{s \neq 0}, s \delta \pm \varepsilon_{i} \pm \varepsilon_{j}, s \delta \pm \varepsilon_{i},(2 s+1) \delta \pm 2 \varepsilon_{i}, s \delta \pm \delta_{g} \pm \delta_{h}, 2 s \delta \pm 2 \delta_{h}\right\}$ \\
& & $\hat{\Delta}_{\overline{1}}=\left\{s \delta \pm \delta_{g} \pm \varepsilon_{i}, s \delta \pm \delta_{g}\right\}$ \\
\hline$A(2 l, 2 k-1)^{(2)}$ & $k \geq l+1$ & $\hat{\Delta}_{\overline{0}}=\left\{s \delta_{s \neq 0}, s \delta \pm \delta_{g} \pm \delta_{h}, s \delta \pm \delta_{g},(2 s+1) \delta \pm 2 \delta_{g}, s \delta \pm \varepsilon_{i} \pm \varepsilon_{j}, 2 s \delta \pm 2 \varepsilon_{i}\right\}$ \\
& & $\hat{\Delta}_{\overline{1}}=\left\{s \delta \pm \varepsilon_{j} \pm \delta_{g}, s \delta \pm \varepsilon_{i}\right\}$ \\
\hline$A(2 k-1,2 l-1)^{(2)}$ & $k \geq l+1$ & $\hat{\Delta}_{\overline{0}}=\left\{s \delta_{s \neq 0}, s \delta \pm \varepsilon_{i} \pm \varepsilon_{j}, s \delta \pm \delta_{g} \pm \delta_{h}, 2 s \delta \pm 2 \delta_{g},(2 s+1) \delta \pm 2 \varepsilon_{i}\right\}$ \\
& & $\hat{\Delta}_{\overline{1}}=\left\{s \delta \pm \varepsilon_{i} \pm \delta_{g}\right\}$ \\
\hline$A(2 l-1,2 k-1)^{(2)}$ & $k \geq l$ & $\hat{\Delta}_{\overline{0}}=\left\{s \delta_{s \neq 0}, s \delta \pm \delta_{g} \pm \delta_{h}, s \delta \pm \varepsilon_{i} \pm \varepsilon_{j}, 2 s \delta \pm 2 \varepsilon_{i},(2 s+1) \delta \pm 2 \delta_{g}\right\}$ \\
& & $\hat{\Delta}_{\overline{1}}=\left\{s \delta \pm \varepsilon_{i} \pm \delta_{g}\right\}$ \\
\hline$A(2 k, 2 l)^{(4)}$ & $k \geq l+1$ & $\hat{\Delta}_{\overline{0}}=\left\{2 s \delta_{s \neq 0}, 2 s \delta \pm \varepsilon_{i} \pm \varepsilon_{j}, 2 s \delta \pm \varepsilon_{i},(4 s+2) \delta \pm 2 \varepsilon_{i}\right.$, \\
& & $\left.2 s \delta \pm \delta_{h} \pm \delta_{h},(2 s+1) \delta \pm \delta_{g}, 4 s \delta \pm 2 \delta_{g}\right\}$ \\
& & $\hat{\Delta}_{\overline{1}}=\left\{(2 s+1) \delta,(2 s+1) \delta \pm \varepsilon_{i}, 2 s \delta \pm \delta_{g}, 2 s \delta \pm \varepsilon_{i} \pm \delta_{g}\right\}$ \\
\hline$A(2 l, 2 k)^{(4)}$ & $k \geq l$ & $\hat{\Delta}_{\overline{0}}=\left\{2 s \delta_{s \neq 0}, 2 s \delta \pm \delta_{g} \pm \delta_{h}, 2 s \delta \pm \delta_{g},(4 s+2) \delta \pm 2 \delta_{g}\right.$, \\
& & $\left.2 s \delta \pm \varepsilon_{i} \pm \varepsilon_{j},(2 s+1) \delta \pm \varepsilon_{i}, 4 s \delta \pm 2 \varepsilon_{j}\right\}$ \\
& & $\hat{\Delta}_{\overline{1}}=\left\{(2 s+1) \delta,(2 s+1) \delta \pm \delta_{g}, 2 s \delta \pm \varepsilon_{i}, 2 s \delta \pm \delta_{g} \pm \varepsilon_{i}\right\}$ \\
\hline$C(l+1)^{(2)}, D(k+1, l)^{(2)}$ & $k \geq l+1$ & $\hat{\Delta}_{\overline{0}}=\left\{s \delta_{s \neq 0}, 2 s \delta \pm \varepsilon_{i} \pm \varepsilon_{j}, 2 s \delta \pm \delta_{g} \pm \delta_{h}, 2 s \delta \pm 2 \delta_{g}, s \delta \pm \varepsilon_{i}\right\}$ \\
& & $\hat{\Delta}_{\overline{1}}=\left\{2 s \delta \pm \varepsilon_{i} \pm \delta_{g}, s \delta \pm \delta_{g}\right\}$ \\
\hline$C(l+1)^{(2)}, D(l+1, k)^{(2)}$ & $k \geq l$ & $\hat{\Delta}_{\overline{0}}=\left\{s \delta_{s \neq 0}, 2 s \delta \pm \delta_{g} \pm \delta_{h}, 2 s \delta \pm \varepsilon_{i} \pm \varepsilon_{j}, 2 s \delta \pm 2 \varepsilon_{i}, s \delta \pm \delta_{g}\right\}$ \\
& & $\hat{\Delta}_{\overline{1}}=\left\{2 s \delta \pm \delta_{g} \pm \varepsilon_{i}, s \delta \pm \varepsilon_{i}\right\}$ \\
\hline$G(3)^{(2)}$ & & $\hat{\Delta}_{\overline{0}}=\left\{2 s \delta_{s \neq 0}, 2 s \delta \pm 2 \varepsilon_{1}, 2 s \delta \pm 2 \varepsilon_{2}, 2 s \delta \pm 2 \varepsilon_{3},(2 s+1) \delta \pm\left(3 \varepsilon_{2}+\varepsilon_{1}\right)\right.$, \\
& $\left.(2 s+1) \delta \pm\left(3 \varepsilon_{2}-\varepsilon_{1}\right),(2 s+1) \delta \pm\left(\varepsilon_{1}+\varepsilon_{2}\right),(2 s+1) \delta \pm\left(\varepsilon_{2}-\varepsilon_{1}\right)\right\}$ \\
& & $\hat{\Delta}_{\overline{1}}=\left\{2 s \delta \pm \varepsilon_{1} \pm \varepsilon_{2} \pm \varepsilon_{3},(2 s+1) \delta \pm 2 \varepsilon_{2} \pm \varepsilon_{3},(2 s+1) \delta \pm \varepsilon_{3}\right\}$ \\
\hline & &
\end{tabular}

TABle 2. Root system types

\begin{tabular}{cccc}
\hline The algebra & Finite Part & $\hat{\Delta}^{\prime}$ & $\hat{\Delta}^{\prime \prime}$ \\
\hline$A(2 k, 2 l-1)^{(2)}, k \geq l$ & $B(k, l)$ & $A_{2 k}^{(2)}$ & $A_{2 l-1}^{(2)}$ \\
\hline$A(2 l, 2 k-1)^{(2)}, k \geq l+1$ & $B(l, k)$ & $A_{2 k-1}^{(2)}$ & $A_{2 l}^{(2)}$ \\
\hline$A(2 k-1,2 l-1)^{(2)}, k \geq l$. & $D(k, l)$ & $A_{2 k-1}^{(2)}$ & $A_{2 l-1}^{(2)}$ \\
\hline$A(2 l-1,2 k-1)^{(2)}, k \geq l$. & $D(l, k)$ & $A_{2 k-1}^{(2)}$ & $A_{2 l-1}^{(2)}$ \\
\hline$A(2 k, 2 l)^{(4)}, k \geq l+1$ & $B(k, l)$ & $A_{2 k}^{(2)}$ & $A_{2 l}^{(2)}$ \\
\hline$A(2 l, 2 k)^{(4)}, k \geq l$ & $B(l, k)$ & $A_{2 k}^{(2)}$ & $A_{2 l}^{(2)}$ \\
\hline$D(k+1, l)^{(2)}, k \geq l$ & $B(k, l)$ & $D_{k+1}^{(2)}$ & $C_{l}^{(1)}$ \\
\hline$D(l+1, k)^{(2)}, k \geq l+1$ & $B(l, k)$ & $C_{k}^{(1)}$ & $D_{l+1}^{(2)}$ \\
\hline$G(3)^{(2)}$ & $D\left(1,2,-\frac{3}{4}\right)$ & $G_{2}^{(1)}$ & $A_{1}^{(1)}$ \\
\hline
\end{tabular}


TABLE 3. Choices of simple roots

\begin{tabular}{|c|c|c|}
\hline The algebra & & Choice of simple roots \\
\hline$A(2 k, 2 l-1)^{(2)}$ & $k \geq l+1$ & $\begin{array}{l}\varepsilon_{1}-\delta_{1}, \delta_{1}-\varepsilon_{2}, \ldots, \varepsilon_{l}-\delta_{l}, \delta_{l}-\varepsilon_{l+1}, \\
\varepsilon_{l+1}-\varepsilon_{l+2}, \ldots, \varepsilon_{k-1}-\varepsilon_{k}, \varepsilon_{k}, \delta-2 \varepsilon_{1}\end{array}$ \\
\hline$A(2 k, 2 k-1)^{(2)}$ & & $\delta_{1}-\varepsilon_{1}, \ldots, \delta_{k}-\varepsilon_{k}, \varepsilon_{k}, \delta-\varepsilon_{1}-\delta_{1}$ \\
\hline$A(2 l, 2 l+1)^{(2)}$ & & $\varepsilon_{1}-\delta_{1}, \ldots, \varepsilon_{l}-\delta_{l}, \delta_{l}-\varepsilon_{l+1}, \varepsilon_{l+1}, \delta-\delta_{1}-\varepsilon_{1}$ \\
\hline$A(2 l, 2 k-1)^{(2)}$ & $k \geq l+2$ & $\begin{array}{l}\varepsilon_{1}-\varepsilon_{2}, \varepsilon_{2}-\delta_{1}, \delta_{1}-\varepsilon_{3}, \ldots, \varepsilon_{l+1}-\delta_{l}, \delta_{l}-\varepsilon_{l+2} \\
\varepsilon_{l+2}-\varepsilon_{l+3}, \ldots, \varepsilon_{k-1}-\varepsilon_{k}, \varepsilon_{k}, \delta-\varepsilon_{1}-\varepsilon_{2}\end{array}$ \\
\hline$A(2 k-1,2 l-1)^{(2)}$ & $k \geq l+2$ & $\begin{array}{l}\varepsilon_{1}-\delta_{1}, \delta_{1}-\varepsilon_{2}, \ldots, \varepsilon_{l-1}-\delta_{l-1}, \delta_{l}-\varepsilon_{l+1} \\
\varepsilon_{l+1}-\varepsilon_{l+2}, \ldots \varepsilon_{k-1}-\varepsilon_{k}, \varepsilon_{k-1}+\varepsilon_{k}, \delta-2 \varepsilon_{1}\end{array}$ \\
\hline$A(2 l+1,2 l-1)^{(2)}$ & & $\varepsilon_{1}-\delta_{1}, \delta_{1}-\varepsilon_{2}, \ldots, \varepsilon_{l-1}-\delta_{l-1}, \delta_{l}-\varepsilon_{l+1}, \delta_{l}+\varepsilon_{l+1}, \delta-2 \varepsilon_{1}$ \\
\hline$A(2 k-1,2 k-1)^{(2)}$ & & $\varepsilon_{1}-\delta_{1}, \delta_{1}-\varepsilon_{2}, \ldots, \varepsilon_{k}-\delta_{k}, \varepsilon_{k}+\delta_{k}, \delta-\varepsilon_{1}-\delta_{1}$ \\
\hline$A(2 l-1,2 l+1)^{(2)}$ & & $\varepsilon_{1}-\varepsilon_{2}, \varepsilon_{2}-\delta_{1}, \delta_{1}-\varepsilon_{3}, \ldots, \varepsilon_{l+1}-\delta_{l}, \varepsilon_{l+1}+\delta_{l}, \delta-\varepsilon_{1}-\varepsilon_{2}$ \\
\hline$A(2 l-1,2 k-1)^{(2)}$ & $k \geq l+2$ & $\begin{array}{l}\varepsilon_{1}-\varepsilon_{2}, \varepsilon_{2}-\delta_{1}, \delta_{1}-\varepsilon_{3}, \ldots, \varepsilon_{l+1}-\delta_{l}, \delta_{l}-\varepsilon_{l+2} \\
\varepsilon_{l+2}-\varepsilon_{l+3}, \ldots \varepsilon_{k-1}-\varepsilon_{k}, 2 \varepsilon_{k}, \delta-\varepsilon_{1}-\varepsilon_{2}\end{array}$ \\
\hline$A(2 k, 2 l)^{(4)}$ & $k \geq l+1$ & $\begin{array}{l}\varepsilon_{1}-\delta_{1}, \delta_{1}-\varepsilon_{2}, \ldots, \varepsilon_{l-1}-\delta_{l}, \delta_{l}-\varepsilon_{l} \\
\varepsilon_{l}-\varepsilon_{l+1}, \ldots \varepsilon_{k-1}-\varepsilon_{k}, \varepsilon_{k}, \delta-\varepsilon_{1}\end{array}$ \\
\hline$A(2 k, 2 k)^{(4)}$ & & $\varepsilon_{1}-\delta_{1}, \delta_{1}-\varepsilon_{2}, \ldots, \varepsilon_{k}-\delta_{k}, \delta_{k}, \delta-\varepsilon_{1}$ \\
\hline$A(2 l, 2 k)^{(4)}$ & $k \geq l+1$ & $\begin{array}{l}\varepsilon_{1}-\delta_{1}, \delta_{1}-\varepsilon_{2}, \ldots, \varepsilon_{l}-\delta_{l}, \delta_{l}-\varepsilon_{l+1} \\
\varepsilon_{l}-\varepsilon_{l+1}, \ldots \varepsilon_{k-1}-\varepsilon_{k}, \varepsilon_{k}, \delta-\varepsilon_{1}\end{array}$ \\
\hline$C(l+1)^{(2)}, D(k+1, l)^{(2)}$ & $k \geq l+1$ & $\begin{array}{l}\varepsilon_{1}-\delta_{1}, \delta_{1}-\varepsilon_{2}, \ldots, \varepsilon_{l}-\delta_{l}, \delta_{l}-\varepsilon_{l+1}, \\
\varepsilon_{l+1}-\varepsilon_{l+2}, \ldots, \varepsilon_{k-1}-\varepsilon_{k}, \varepsilon_{k}, \delta-\varepsilon_{1}\end{array}$ \\
\hline$D(k+1, k)^{(2)}$ & & $\varepsilon_{1}-\delta_{1}, \delta_{1}-\varepsilon_{2}, \ldots, \varepsilon_{k}-\delta_{k}, \delta_{k}, \delta-\varepsilon_{1}$ \\
\hline$C(l+1)^{(2)}, D(l+1, k)^{(2)}$ & $k \geq l+1$ & $\begin{array}{l}\varepsilon_{1}-\delta_{1}, \delta_{1}-\varepsilon_{2}, \ldots, \varepsilon_{l}-\delta_{l}, \delta_{l}-\varepsilon_{l+1}, \\
\varepsilon_{l+1}-\varepsilon_{l+2}, \ldots, \varepsilon_{k-1}-\varepsilon_{k}, \varepsilon_{k}, \delta-\varepsilon_{1}\end{array}$ \\
\hline$G(3)^{(2)}$ & & $\varepsilon_{3}-\varepsilon_{2}-\varepsilon_{1}, 2 \varepsilon_{1}, 2 \varepsilon_{2}, \delta-\left(\varepsilon_{3}+2 \varepsilon_{2}\right)$ \\
\hline
\end{tabular}

\section{REFERENCES}

[FKP] Victor G. Kac, Pierluigi Moseneder Frajria, Paolo Papi, Denominator identities for finite-dimensional Lie superalgebras and Howe duality for compact dual pairs, arXiv:1102.3785.

[G1] M. Gorelik, Weyl denominator identity for affine Lie superalgebras with non-zero dual Coxeter Number, J. Algebra, 337(1), (2011), 50-62.

[G2] M. Gorelik, Weyl denominator identity for finite-dimensional Lie superalgebras, to appear in "Highlights in Lie Theory", Progress in Math., Birkhauser Boston, MA.

[G3] M. Gorelik, Weyl denominator identity for the affine Lie superalgebras $\widehat{\mathfrak{g l}}(2 \mid 2)$, arXiv:1007.4305

[GR] M. Gorelik, S. Reif, Denominator identity for affine Lie superalgebras with zero dual Coxeter number, arXiv: 1012.5879

[GK] M. Gorelik, V.G. Kac, On Simplicity of Vacuum modules, Advances in Math, 211 (2007), 621-677.

[HR] C. Hoyt, S. Reif, Simplicity of vacuum modules over affine Lie superalgebras, J. Algebra 321, No. 10, (2009), 2861-2874.

[HS] C. Hoyt, V. Serganova, Classification of finite growth general Kac-Moody superalgebras and integrability, Comm. in Algebra, 35, (2007), 851-874.

[K] V.G. Kac, Infinite dimensional Lie algebras, Cambridge University Press, 1990.

[KW] V.G. Kac, M. Wakimoto, Integrable highest weight modules for affine Lie superalgebras and number theory, in Lie Theory and Geometry, 415-456, Progress in Math., 123, Birkhauser Boston, MA, 1994.

[M] S. Milne, Infinite families of exact sums of squares formulas, Jacobi elliptic functions, continued fractions, and Schur functions, Ramanujan J. 6 (2002), 7-149. 
[S] V. Serganova, Kac-Moody superalgebras and integrability, Developments and Trends in InfiniteDimensional Lie Theory, Progr. Math. vol. 288, Birkhäuser Boston, Inc., Boston, MA (2011), pp. 169-218.

[vdL1] J. W. van de Leur, A classification of Contragredient Lie superalgebras, Comm. in Algebra 17(8) (1989), 1815-184.

[vdL2] J. W. van de Leur, Contragredient Lie Superalgebras of Finite Growth, Ph.D. Thesis, 1986.

[Z] D. Zagier, A proof of the Kac-Wakimoto affine denominator formula for the strange series, Math. Res. Lett. 7 (2000), no. 5-6, 597-604. 